\title{
Severe covid-19 pneumonia: pathogenesis and clinical management
}

\author{
Amy H Attaway, ${ }^{1}$ Rachel G Scheraga, ${ }^{1,2}$ Adarsh Bhimraj, ${ }^{1}$ Michelle Biehl, ${ }^{1}$ Umur Hatipoğlu ${ }^{1}$
}

Check for updates

${ }^{1}$ Respiratory Institute, Cleveland Clinic, Cleveland, Ohio, USA

${ }^{2}$ Inflammation and Immunity, Cleveland Clinic, Cleveland,

Ohio, USA

Correspondence to:

A Attaway

attawaa@ccf.org

Cite this as: $B M J$ 2021;372:n436 http://dx.doi.org/10.1136/bmj.n436

Series explanation: State of the Art Reviews are commissioned on the basis of their relevance to academics and specialists in the US and internationally. For this reason they are written predominantly by US authors.

\begin{abstract}
Severe covid-19 pneumonia has posed critical challenges for the research and medical communities. Older age, male sex, and comorbidities increase the risk for severe disease. For people hospitalized with covid-19, 15-30\% will go on to develop covid-19 associated acute respiratory distress syndrome (CARDS). Autopsy studies of patients who died of severe SARS CoV-2 infection reveal presence of diffuse alveolar damage consistent with ARDS but with a higher thrombus burden in pulmonary capillaries. When used appropriately, high flow nasal cannula (HFNC) may allow CARDS patients to avoid intubation, and does not increase risk for disease transmission. During invasive mechanical ventilation, low tidal volume ventilation and positive end expiratory pressure (PEEP) titration to optimize oxygenation are recommended. Dexamethasone treatment improves mortality for the treatment of severe and critical covid-19, while remdesivir may have modest benefit in time to recovery in patients with severe disease but shows no statistically significant benefit in mortality or other clinical outcomes. Covid-19 survivors, especially patients with ARDS, are at high risk for long term physical and mental impairments, and an interdisciplinary approach is essential for critical illness recovery.
\end{abstract}

\section{Introduction}

The ongoing outbreak of the coronavirus disease 2019 (covid-19) has posed immense challenges for the research and medical communities. This review focuses on the epidemiologic and clinical features of covid-19, the pathophysiologic mechanisms, inpatient respiratory support, and the evidence to date on drug treatments. It also covers the recovery and long term management of patients with covid-19 pneumonia. The review is aimed at clinicians and intensivists caring for patients with severe covid-19 pneumonia as defined by the National Institutes of Health, ${ }^{1}$ referring to individuals with SARS-CoV-2 infection confirmed by polymerase chain reaction (PCR) testing who have $\mathrm{SpO}_{2}<94 \%$ on room air at sea level, a ratio of arterial partial pressure of oxygen to fraction of inspired oxygen $\left(\mathrm{PaO}_{2} / \mathrm{FiO}_{2}\right)<300 \mathrm{~mm}$ $\mathrm{Hg}$, respiratory frequency $>30$ breaths/min, or lung infiltrates $>50 \%$.

\section{Methods}

We manually searched electronic databases PubMed and Embase for English language articles published from 1 January 2020 to 20 February 2021. We also reviewed the medRxiv preprint server to monitor the rapidly evolving information on covid-19. We used the following search terms in combination with the term "covid-19": "pneumonia", "ARDS", "pathogenesis”, "epidemiology”, "survival”,

\section{ABBREVIATIONS}

RCTs (randomized controlled trials), OR (odds ratio), ECMO (extra corporeal membrane oxygenation), RR (rate ratio), HFNC (high flow nasal cannula), NIV (non-invasive ventilation), IMV (invasive mechanical ventilation), HCQ (hydroxychloroquine), CP (convalescent plasma), EUA (emergency use authorization), ED (emergency department), IV (intravenous), PICS (post-intensive care syndrome), ICU (intensive care unit), ARDS (acute respiratory distress syndrome), MoCA (Montreal Cognitive Assessment), MRI (magnetic resonance imaging), IQR (interquartile range), $\mathrm{PEEP}$ (positive end expiratory pressure), PPE (personal protective equipment), NMB (neuromuscular blockade), CARDS (coronavirus associated acute respiratory distress syndrome), Pplat (plateau pressure), COPD (chronic obstructive pulmonary disease), CHF (congestive heart failure), SARS-CoV-2 (severe acute respiratory syndrome coronavirus 2), NYC (New York City), IES-R (impact of event scale-revised), EQ-5D (European Quality of Life Five Dimension), HADS (Hospital Anxiety and Depression Scale), ADL (activities of daily living), iADL (instrumental activities of daily living)

"therapeutics", and “complications". We included articles on the basis of the quality of the study and 
favored large randomized controlled trials (RCTs), high quality observational studies, systematic reviews, meta-analyses, and guidelines. Because of the evolving nature of the pandemic, the paucity of data, and the lack of RCTs, our article selection for respiratory care and post-covid complications included observational studies and case series. We excluded case reports and articles in non-peer reviewed journals.

\section{Clinical manifestations and epidemiology}

At the time of writing, covid-19 is responsible for 116 million cases globally and 2.5 million deaths. ${ }^{2}$ The most striking characteristic of the disease is its heterogeneity, ranging from no symptoms to critical illness. ${ }^{3}$ Older age, male sex, race (particularly Black, Hispanic, and South Asian), and comorbidities (including hypertension, diabetes, cardiovascular disease, chronic pulmonary disease, chronic kidney disease, cancer, and chronic liver disease) have been associated with worse outcomes. ${ }^{3-8}$ Genetic factors may play a part as well, with blood type A associated with a higher risk for severe disease. ${ }^{9}$ A common characteristic of SARS-CoV-2 is asymptomatic transmission, ${ }^{10}$ which is likely the cause of rampant spread and transmission. ${ }^{11}$ Given SARS-CoV-2 entry is primarily via the respiratory tract, upper and lower respiratory tract involvement is the most common manifestation. ${ }^{12}$ About one third of patients hospitalized with SARS-CoV-2 infection meet criteria for acute respiratory distress syndrome. ${ }^{13}$ In-hospital mortality, while initially very high in certain series $(60 \%$ for those intubated in a large study from New York City in April 2020 ${ }^{14}$ ) has been declining during the course of the pandemic, with in-hospital survival improving from $74.4 \%$ (March 2020) to 92.4\% (August) in a study from New York City, ${ }^{15}$ and intensive care unit (ICU) survival improving from $58 \%$ (March) to $80 \%$ (June) in a large national surveillance database from England. ${ }^{16}$

\section{Pathophysiologic mechanisms}

Structure of SARS-CoV-2

SARS-CoV-2 is a positive sense, single stranded RNA enveloped virus in the Betacoronavirus genus. ${ }^{12} 17$ Bats and pangolins may be the animal hosts of SARS-CoV-2 as there is a $>90 \%$ gene homology to the SARS-CoV-2 found to infect humans. ${ }^{12}{ }^{18}$ Currently it remains unclear if SARS-CoV-2 was directly transferred from bat/pangolins to humans or an intermediate host was required for transmission. ${ }^{12} 18$ In light of the current pandemic, researchers first compared SARS-CoV-2 with the previous endemic SARS-CoV (2002-03) and MERS-CoV (2012). ${ }^{19}$ SARSCoV-2 has overlapping genetic sequences with SARS$\mathrm{CoV}$ and MERS-CoV, with 79\% and 50\% homology, respectively. $^{172021}$

SARS-CoV-2 is characterized by four main structural proteins that are important for infectivity and replication. ${ }^{20}$ These proteins include the spike $(\mathrm{S})$, membrane $(\mathrm{M})$, envelope (E), and nucleocapsid (N) proteins. ${ }^{22} 23$ The $\mathrm{S}$ protein, which includes two protein subunits (S1 and S2), gives the virus its well known appearance as the $\mathrm{S}$ protein protrudes from the membrane. ${ }^{24}$ The tip of the protruding $\mathrm{S}$ protein has a crown (Latin corona)-like shape. ${ }^{24}$ The $\mathrm{S}$ protein is also important for binding to the angiotensin converting enzyme 2 (ACE2) receptor, which is the point of entry of the virus to the human and animal host. ${ }^{25}$ Furthermore, the S protein is thought to be a major contributor to the immunogenic response; therefore the $S$ protein is the target of most vaccines. $^{25} 26$ The $M$ protein is a transmembrane protein important in viral pathogenesis. ${ }^{27}$ Little is understood about the E protein; however, it is known to play a role in viral replication and infectivity. 2829 Finally, the $\mathrm{N}$ protein allows for regulation of viral RNA replication, transcription, and synthesis. ${ }^{30}$

\section{SARS-CoV-2 mutations}

Emerging data show distinctive mutations in the SARS-CoV-2 genome isolated from patients. ${ }^{31}$ SARSCoV-2 mutated variants include B.1.1.7 (UK variant), P.1 (Brazilian variant) ${ }^{32}$, and B.1.351 (South African variant). ${ }^{33}$ The primary region of mutation for these variants is in the spike protein. The B.1.1.7 variant has a greater rate of infectivity and spread, ${ }^{32}$ which may be related to binding affinity to the ACE2 receptor. $^{34}$

SARS-CoV-2 invasion and replication in cells (fig 1) Early knowledge of the entry process of SARS-CoV-2 into host cells, via the binding of the $S$ protein to the ACE2 receptor, was extrapolated from what was known from SARS-CoV. ${ }^{35} 36$ Human ACE2 (hACE) receptor is the same receptor used by SARS-CoV for viral entry. ${ }^{37}$ hACE receptor is similar across animal species but with a varied binding efficiency. ${ }^{37}$ Older age and male sex of the host are also determinants of S protein-ACE2 binding efficiency. ${ }^{38}$ ACE2 receptors are highly expressed in the upper respiratory tract of humans. ${ }^{17}$ Proteolytic cleavage of the S protein by serine proteases including transmembrane protease serine 2 (TMPRSS2), cathepsin L, and furin, are required for binding to the ACE2 receptor. ${ }^{35}$ Similar to the ACE2 receptor, protease expression varies by tissue type and location, with a high expression in the nasal and bronchial epithelium. ${ }^{39}$ In addition, human epithelial cells that line mucosal surfaces and cover organs such as conjunctiva, gastrointestinal tract, liver, and kidney also express ACE2 and TMPRSS2. ${ }^{40} 41$ Once the virus attaches to the host cell receptors, it undergoes endocytosis, viral maturation, replication, and release of more virus within the cytoplasm of the host cell. ${ }^{37}$ SARS-CoV-2 infection begins with viral replication and partially avoids host recognition during the initial infection and before the host innate response is enabled. ${ }^{42}$

\section{Host response}

Limited mechanistic data are available on the innate immune response to SARS-CoV- $2^{42}$ although expansion of in vitro studies, animal models, and covid-19 patient serum profiles has been 


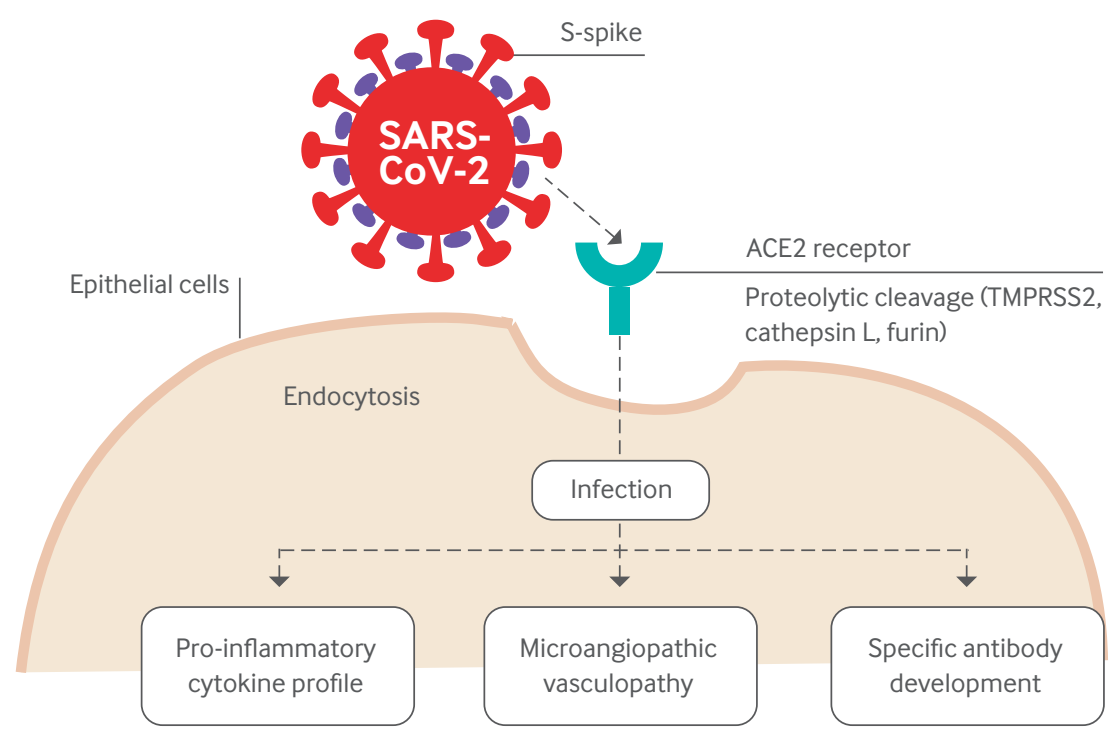

Fig 1 | SARS-CoV-2 S spike protein binds to the ACE2 receptor, which leads to proteolytic cleavage by TMPRSS2, cathepsin L, and furin in the epithelial cell of the respiratory tract. The virus undergoes endocytosis, viral maturation, replication, and release of more virus within the cytoplasm infecting the host cell. Consequences of infected cells include pro-inflammatory cytokine secretion, microangiopathic vasculopathy, and B cell secretion of specific SARSCoV-2 antibodies

significant. ${ }^{43}$ It is now evident that over the first few days after SARS-CoV infection, activation of toll-like receptors (TLR 3, 7, and 8) by pathogen recognition receptors (PRRs) induces transcriptional upregulation of interferons (type I and III interferons) and recruitment of leukocytes. ${ }^{43}$

The magnitude of the innate antiviral response has been associated with the degree of infection, which might account for the heterogeneous viral response among those infected with covid-19..$^{42}$ The adaptive immune response starts with IgA, IgG, and IgM specific antibody release similar to the response to SARS-CoV. ${ }^{44}$ The timing of antibody release and the persistence of detectable levels has varied among patients..$^{44}$ Case and observational studies in patients with SARS-CoV-2 showed early detection of specific IgA and IgM antibodies (within five days) and late detection of specific IgG antibodies (after 14 days). ${ }^{44}$ In addition, disease severity has recently been shown to drive an enhanced antibody response, ${ }^{45} 46$ which correlates with clinical outcomes. ${ }^{47}$

Clinical observation of lymphopenia has been apparent since the start of the covid-19 pandemic and may be associated with worsening disease. ${ }^{48}$ An adequate $\mathrm{T}$ cell response (both $\mathrm{CD}^{+}$and $\mathrm{CD}^{+} \mathrm{T}$ cells) directed toward SARS-CoV-2 has been shown to be associated with milder disease. ${ }^{49}$ Aging is well established to be associated with failure of regeneration of naive $\mathrm{T}$ cells and $\mathrm{T}$ cell activation. ${ }^{50}$ In covid-19, dysregulation of $\mathrm{T}$ cell homeostasis has been postulated as a mechanism for severe disease seen in older adults. ${ }^{49}$ Direct anti-SARS-CoV-2 antibodies have been manufactured for treatment by Regeneron (REGN10933 and REGN 10987) and Eli Lilly (LY-CoV016) to bind to the viral receptor binding domain. ${ }^{34}$ Concern is ongoing that the mutations would give the virus the ability to escape direct binding to the specific antibodies. ${ }^{34}$ More research is needed to fully identify the impact the virus mutations have on the treatment modalities available.

Early descriptions of covid-19 included development of a cytokine storm as a harbinger for clinical deterioration. ${ }^{51}$ Clinical and serologic evidence points to high levels of serum IL-6, IL-1 $\beta$, and TNF- $\alpha$ which are associated with clinical instability and other biomarkers of inflammation. ${ }^{52-54}$ More recent studies comparing serum cytokine measurements with other known cytokine mediated diseases such as sepsis and cytokine release syndrome have noted that covid-19 patients' serum cytokine levels were substantially lower. $^{515255}$ As a result, the direct role of cytokines in disease pathogenesis has been challenged. ${ }^{55}$ Many unanswered questions related to the pathogenesis of inflammation and the mechanism of action of corticosteroids in covid- 19 .

Autopsy studies of patients who have died from severe SARS CoV-2 infection reveal presence of alveolar wall injury and diffuse alveolar damage consistent with ARDS. ${ }^{567}$ However, compared with classic ARDS, autopsy studies also indicate higher thrombus burden in pulmonary capillaries, which suggests a greater pathogenic role of thrombotic and microangiopathic vasculopathy in covid-19 related ARDS..$^{56} 57$ Studies collectively show that thromboembolism occurs more frequently and is associated with a higher mortality in patients with covid-19. ${ }^{58} 59$ Additional studies are needed to delineate the direct clinical consequences of increased thrombosis and its association with mortality in covid-19, which have major implications for the management of respiratory failure. Current 
studies are ongoing to investigate treatment with anticoagulants, which may shed light on the importance of thrombosis in covid-19 ARDS.

\section{Respiratory care for severe covid-19 pneumonia}

Severe covid-19 pneumonia as defined by $\mathrm{NIH}^{1}$ overlaps significantly with the clinical definition of "classic" ARDS. ${ }^{60}$ However, several unique pathophysiological processes are postulated to be at play for CARDS, such as intravascular thrombosis caused by loss of endothelial barrier, prominent loss of hypoxic pulmonary vasoconstriction resulting from endothelial dysfunction, and excessive blood flow to collapsed lung tissue. ${ }^{61}$ Further, not all case series provide a clear semantic distinction between severe covid-19 pneumonia and CARDS, which confounds interpretation. In this section, we summarize the current literature on the use of respiratory therapy equipment in patients with severe covid-19 pneumonia. To date, no controlled prospective trials inform the respiratory management of severe covid-19 pneumonia. Notwithstanding, among patients with severe covid-19 pneumonia, patient respiratory system mechanics and clinical outcomes achieved with standard ARDS management are similar to classic ARDS. Consequently, contemporary respiratory care revolves around supportive measures and is based on the management of classic ARDS. We begin by providing a general review of these concepts.

Titration of oxygen therapy to avoid hyperoxemia $^{6263}$ and hypoxemia ${ }^{64}$ is strongly recommended for acute hypoxemic respiratory failure. A range of $90-96 \%$ oxygen saturation, confirmed by cooximetry, is a reasonable target. ${ }^{63}$ For patients who require invasive mechanical ventilation (IMV), the first goal is avoidance of high tidal volumes, which are associated with ventilator induced lung injury. ${ }^{65} 66$ Evidence suggests that similar injury could occur because of sustained high tidal volumes during spontaneous breathing, also known as patient self-induced lung injury (P-SILI). ${ }^{67-69}$ Although not validated in controlled clinical trials, an assessment of strain known as tidal pressure or driving pressure ${ }^{70}$ (defined as the ratio of tidal volume to tidal respiratory system compliance) allows matching of volume delivery with respiratory system mechanics and enables optimal mechanical ventilatory settings. In an observational study of non-covid ARDS trials, mediation analysis revealed that $75 \%$ of the beneficial effect of treatment group assignment was attributable to reduction in tidal pressure. $^{70}$

The second goal of mechanical ventilation in ARDS is to prevent the constant opening and closing of alveoli which may be injurious to the lung (atelectrauma). Positive end expiratory pressure (PEEP) is titrated to keep alveolar units open throughout the respiratory cycle. Several RCTs that aimed to optimize recruitment in the intervention arm showed similar clinical outcomes to controls ${ }^{72} 73$ and a signal for potential harm which was attributed to recruitment maneuvers. ${ }^{74}$ To that end, the benefits of higher PEEP are evident only when reducing tidal pressure-ie, less strain for a given tidal volume. ${ }^{70}$ Recruitability (the ability to open and keep alveoli open) can be assessed at the bedside by calculating the recruitment/inflation (R/I) ratio. ${ }^{7576}$ For patients who are proven recruitable, employing the high PEEP and $\mathrm{F}_{\mathrm{i}} \mathrm{O}_{2}$ table $^{72}$ may be preferable while monitoring cardiac output and respiratory mechanics to avoid concurrent hyperinflation. ${ }^{77} 78$

Prone ventilation and neuromuscular blockade (NMB) are frequent adjuncts in the treatment of ARDS. Prone ventilation promotes lung recruitment and improves ventilation/perfusion matching by creating a more even distribution of transpulmonary pressure throughout the chest. A multicenter, prospective RCT showed that among patients with severe hypoxemic respiratory failure $\left(\mathrm{P}_{\mathrm{a}} \mathrm{O}_{2} / \mathrm{F}_{\mathrm{i}} \mathrm{O}_{2}\right.$ $<150$ ), prone positioning $>16$ hours a day was associated with reduced 28 day mortality. ${ }^{79} \mathrm{NMB}$ in early ARDS potentially reduces lung strain by eliminating spontaneous breathing activity. Despite earlier encouraging findings, a recent meta-analysis of five RCTs showed no mortality benefit, with a modest reduction in barotrauma risk and improved oxygenation if applied after 48 hours in patients with severe ARDS. ${ }^{80}$

The belief that respiratory care principles to treat classic ARDS should apply in CARDS was challenged when earlier series of covid-19 patients seemed to indicate two different respiratory failure phenotypes. $^{81}$ A case series $(n=16)$ noted that patients had low elastance, low ventilation perfusion matching, low recruitability and lung weight which they named the "L type." Conceivably, such discrepancy of ventilation perfusion matching with relatively normal mechanics was attributed to loss of lung perfusion regulation and hypoxic vasoconstriction. The remainder of the cases were more consistent with classic ARDS (high elastance, high ventilation/perfusion ratio, high recruitability and lung weight) referred to as the " $\mathrm{H}$ type." The authors suggested that patients who had the L type may not require low tidal volume ventilation and attempts at recruitment could bring harm. Further, they reasoned that patients who present with a paucity of infiltrates, low elastance, and hypoxemia should be placed on mechanical ventilation earlier to prevent spontaneous high tidal volumes generated by the patients. This proposed need for a different management has been contested on the grounds of inconclusive evidence for P-SILI and CARDS case series that revealed respiratory system mechanics similar to classic ARDS. ${ }^{82} 83$

Current observational reports mirror our experience and reinforce our view that a significant proportion of patients with covid-19 pneumonia can be treated non-invasively (ie, high flow nasal cannula (HFNC) or non-invasive ventilation (NIV)) in lieu of invasive mechanical ventilation (IMV). This approach may also optimize utilization of mechanical ventilators, a scarce resource during the 
pandemic. We recommend using the entire spectrum of non-invasive and invasive devices for respiratory assistance (fig 2). Figure 2 is based on our practice in treating severe covid-19 pneumonia, and draws largely from the experience in classic ARDS. Close monitoring and attention to signs of non-invasive device failure are crucial for optimal outcomes. Extra corporeal membrane oxygenation (ECMO) is available for patients who have refractory hypoxemia after these measures ${ }^{84}$ but is infrequently needed. ${ }^{85}$

The following sections provide an overview of the different respiratory equipment and outline the rationale for their use in severe covid-19 pneumonia.

\section{High flow nasal cannula oxygen therapy}

HFNC oxygen therapy refers to the delivery of humidified and heated oxygen at high flows, typically $20-60 \mathrm{~L} / \mathrm{min}$, which is titrated to a precise fraction of inspired oxygen $\left(\mathrm{F}_{\mathrm{i}} \mathrm{O}_{2}\right)$. The advantages of delivering oxygen in this manner include improved comfort by satisfying patient flow demand, ${ }^{86}$ creating an oxygen reservoir in the upper airway thereby reducing physiological dead space (reduced $\mathrm{CO}_{2}$ rebreathing), ${ }^{87}$ and providing a modest PEEP that could help recruit collapsed alveoli ${ }^{88}$ with consequent reduction in work of breathing.

Recent meta-analyses suggest that application of HFNC in the setting of acute hypoxemic respiratory failure can reduce the risk of intubation and invasive mechanical ventilation by $15 \%$ compared with conventional oxygen therapy without affecting mortality. ${ }^{8990}$ However, use of HFNC requires vigilant monitoring for signs of impending respiratory failure. Roca and colleagues devised and validated the ROX index (ratio of oxygen saturation by pulse oximetry/ $\mathrm{F}_{\mathrm{i}} \mathrm{O}_{2}$ to respiratory rate) as a bedside tool for predicting HFNC failure in the setting of pneumonia and hypoxemic respiratory failure. ${ }^{91}$ Accordingly, patients with a ROX index $\geq 4.88$ after 2,6 , and 12 hours of treatment had low risk of intubation, whereas a ROX index $<3.85$ at the same time points was associated with a high risk of failure. Delaying intubation until the occurrence of overt desaturation, hypotension, respiratory rate $>35$ breaths/min with respiratory distress, or acidosis has been associated with poor clinical outcomes. ${ }^{92}$

Evidence on the use of HFNC for covid-19 pneumonia consists of case reports and case

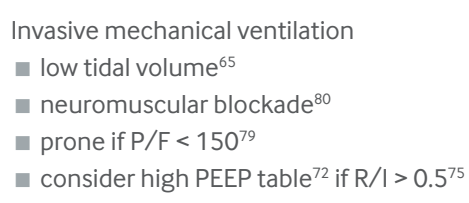

Patient with Severe COVID-19 Pneumonia and Hypoxemic Respiratory Failure

( $\mathrm{SaO} 2<90 \%$ on oxygen mask at $50 \%$ )

Increased Work of Breathing

(accessory muscle use, RR > 30/min, intercostal retractions, abdominal paradox, tracheal tug, nasal flaring)

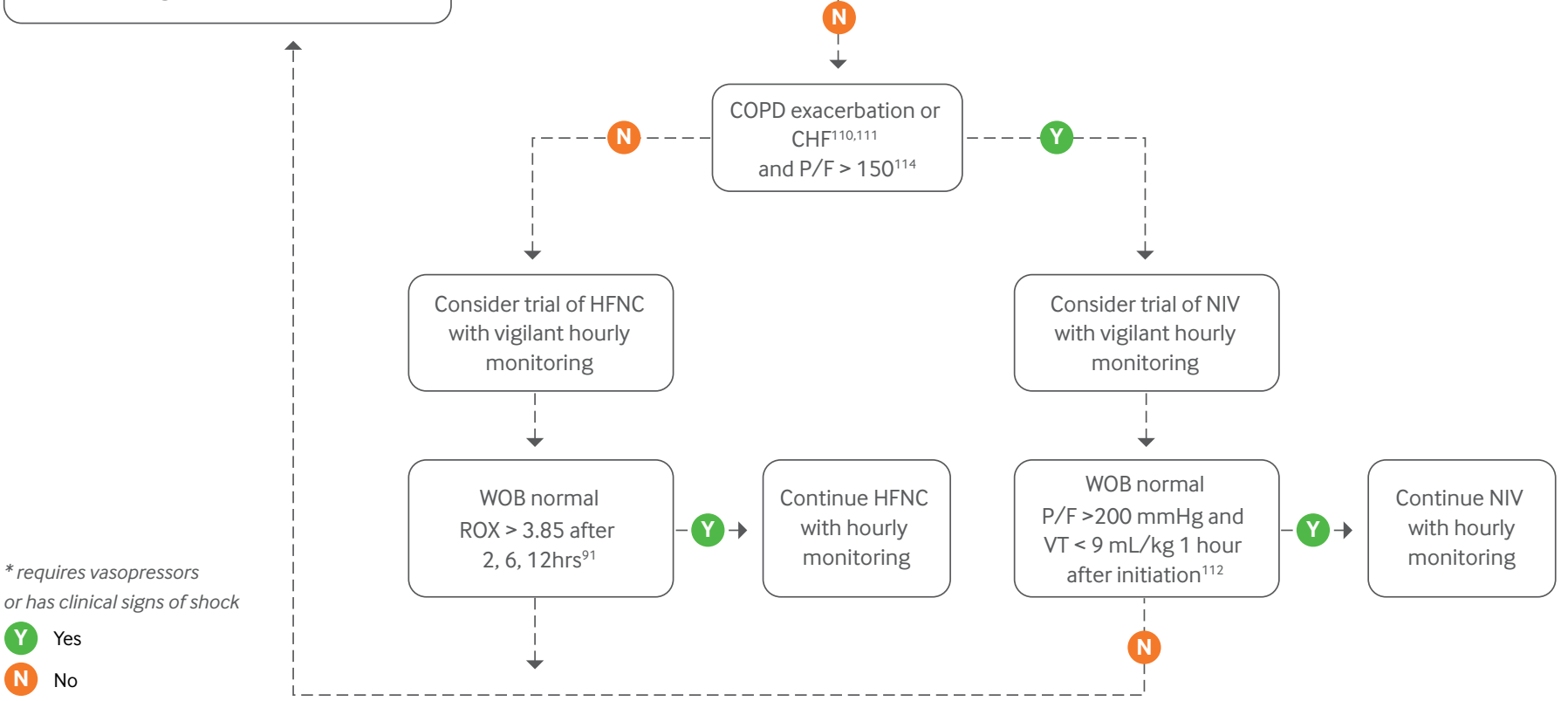

Fig 2 | Algorithm for the respiratory management of a patient with covid-19 pneumonia. RR=respiratory rate, PEEP=positive end expiratory pressure, $\mathrm{R} / \mathrm{I}=$ recruitment/inflation ratio, $\mathrm{COPD}=$ chronic obstructive pulmonary disease, $\mathrm{CHF}=$ congestive heart failure, $\mathrm{HFNC}=\mathrm{high}$ flow nasal cannula, $\mathrm{WOB}=$ work of breathing, $\mathrm{P} / \mathrm{F}=\mathrm{PaO} 2 / \mathrm{FiO} 2$ ratio, $\mathrm{MAP}=$ mean arterial pressure, $\mathrm{NIV}=$ non-invasive ventilation 
series. $^{93-102}$ It attests to the feasibility of utilizing HFNC in this setting; however, firm conclusions regarding efficacy are difficult to draw because of the lack of control groups. Table 1 shows large case series in the English language and provides detailed patient information and outcomes. The patients in these series had respiratory failure with $\mathrm{P}_{\mathrm{a}} \mathrm{O}_{2} / \mathrm{F}_{\mathrm{i}} \mathrm{O}_{2}$ ranging from 68 to 209. The average duration of HFNC was between three and six days; however, patients who required escalation of care did so earlier in the course of treatment. HFNC was associated with successful outcomes (ie, no escalation of care) in 34\% to $70 \%$ of cases. ROX index determined after 4-6 hours of treatment predicted escalation of care. ${ }^{939597}$ Patients with $\mathrm{P}_{\mathrm{a}} \mathrm{O}_{2} / \mathrm{F}_{\mathrm{i}} \mathrm{O}_{2}>200$ before commencing HFNC and who had a reduction in respiratory rate within the first several hours had the best outcomes. ${ }^{101}$ Of note, HFNC treatment is feasible in conjunction with proning patients who have not been intubated (awake proning) and improves oxygenation. However, an observational study noted no difference in the rate of intubation between supine and proned patients. ${ }^{94}$

HFNC was avoided at the beginning of the SARS CoV-2 pandemic in favor of early intubation for fear of disease transmission by exhaled aerosol. However, disease transmission has not been shown in clinical studies. ${ }^{103}$ HFNC does not lead to aerosol generation ${ }^{104} 105$ and aerosol dispersion can be limited by having patients wear masks. ${ }^{106}$ To that end, experts suggest clinicians utilize HFNC treatment for covid-19 patients no differently than for those without infection ${ }^{107}$ with careful attention to proper use of personal protective equipment (PPE). ${ }^{98} 106$ Despite the lack of controlled trials in covid-19, large case series show favorable outcomes for patients who receive therapy with HFNC. A recent computer simulation study concluded that strategies incorporating HFNC for patients not urgently needing intubation could result in greater mechanical ventilator availability and fewer deaths. ${ }^{108}$ Propensity score matched analyses comparing HFNC and other means of respiratory assistance suggest lesser likelihood of intubation, ${ }^{102}$ higher number of ventilator free days and reduction in ICU length of stay ${ }^{109}$ with the former.

\section{Non-invasive ventilation}

Non-invasive ventilation (NIV) is delivered through a face mask or a helmet that is placed over the patient's head. The helmet interface potentially presents a safer alternative (from an infection control perspective) because it eliminates leaks. In the settings of acute congestive heart failure and acute hypercapnic respiratory failure due to COPD, NIV has been extremely effective in preventing intubation and reducing mortality. ${ }^{110} 111$

Application of NIV in the setting of acute hypoxemic respiratory failure excluding COPD and cardiogenic pulmonary edema has been controversial, with mixed results. ${ }^{68112-115}$ Several red flags were raised for NIV when treating ARDS patients. For instance, in the LUNG SAFE study, overall success rate for NIV in classic ARDS was $63 \%$ with an in-hospital mortality of $36 \%$. NIV was associated with higher intensive care unit mortality among ARDS patients with $\mathrm{P}_{\mathrm{a}} \mathrm{O}_{2} /$ $\mathrm{F}_{\mathrm{i}} \mathrm{O}_{2}<150 \mathrm{~mm} \mathrm{Hg}$ on presentation. ${ }^{116}$ A prospective observational study reported failure of NIV in the presence of high expired tidal volumes $(>9.5 \mathrm{~mL} /$ $\mathrm{kg}$ predicted body weight) and poor oxygenation at baseline $\left(\mathrm{P}_{\mathrm{a}} \mathrm{O}_{2} / \mathrm{F}_{\mathrm{i}} \mathrm{O}_{2}<200 \mathrm{mmHg}\right) .{ }^{68}$ Similarly, one hour after initiation of NIV, expired tidal volumes $>9 \mathrm{~mL} / \mathrm{kg}$ of predicted body weight and $\mathrm{P}_{\mathrm{a}} \mathrm{O}_{2} / \mathrm{F}_{\mathrm{i}} \mathrm{O}_{2}$ $\leq 200 \mathrm{mmHg}$ independently predicted NIV failure. ${ }^{112}$ A post hoc analysis reported higher risk of intubation and mortality for patients treated with NIV versus HFNC in a group of immunocompromised patients with acute respiratory failure. ${ }^{117}$ A recent network meta-analysis of 25 RCTs comparing standard oxygen treatment with NIV or HFNC showed lower risk of intubation (HFNC risk ratio 0.76 [95\% confidence interval, 0.55 to 0.99 ]; NIV risk ratio 0.76 [95\% confidence interval, 0.62 to 0.90$]$ ) and lower risk of mortality (NIV risk ratio 0.83 [95\% confidence interval, 0.68 to 0.99]). ${ }^{118}$ However, mortality benefit for NIV delivered by face mask vanished for patients with severe hypoxemia $\left(\mathrm{P}_{\mathrm{a}} \mathrm{O}_{2} / \mathrm{F}_{\mathrm{i}} \mathrm{O}_{2} \leq 200\right)$ when excluding COPD, heart failure, or postoperative patients. In contradistinction, when helmet interface was used to facilitate NIV, the benefit on mortality was maintained, emphasizing the possible importance of how NIV is provided.

A concern with respect to NIV is the higher risk for disease transmission, as noted in previous viral epidemics ${ }^{103}$ because of mask leaks and aerosol dispersion. NIV use was limited in the US and Europe owing to concerns over disease transmission and questionable efficacy in ARDS. ${ }^{119} 120$ In China, on the other hand, NIV was used as the initial strategy between $57 \%$ and $85 \%$ of the time, ${ }^{121-123}$ and to date no clear evidence shows increased disease transmission to healthcare workers. ${ }^{122} 124$

The studies that report detailed patient characteristics and outcomes for the use of NIV in covid-19 pneumonia (table 1) are limited to case series. ${ }^{100} 121123125-129$ Owing to the observational nature of the studies, NIV management is not driven by protocol and no specific guidance is provided on titration of support or when to intubate. Outcome data suffer from incomplete reporting and reveal highly variable hospital survival ranging from 14\% to $95 \%$. $^{125} 129$ Preliminary outcome data from Italy were also not as promising for the use of helmet CPAP in covid-19 as they were for non-covid respiratory failure. ${ }^{125}{ }^{130}$ In a retrospective study ${ }^{125}$ the patients on helmet CPAP died without intubation $54.9 \%$ of the time, attesting to the resource limited conditions under which the study was conducted. Patients with chronic illness, ${ }^{123}$ severe disease on presentation, ${ }^{121}{ }^{130}$ and increased inflammatory markers ${ }^{123129130}$ were at risk for NIV failure. A large prospective single day study from Italy indicated that NIV was successfully used outside of the ICU setting using helmet CPAP in two thirds of the cases of severe covid-19 pneumonia. $^{131}$ A retrospective analysis 
( $n=40$ ) of covid-19 patients who eventually required IMV found that time spent on NIV and HFNC before intubation was associated with higher mortality. ${ }^{132}$ More recent retrospective cohort studies, which employed multivariable risk adjustment, suggest NIV is safe $\mathrm{e}^{133}$ and potentially superior to early intubation $^{134-136}$ strategy. Because thresholds for intubation and clinical monitoring over the course of illness were not standardized a priori, it is difficult to draw firm conclusions from these observational studies.

In the absence of concomitant COPD or pulmonary edema, the benefits of NIV are uncertain in the management of ARDS, and we prefer HFNC as the initial non-invasive support in severely hypoxemic patients with CARDS. When NIV is utilized, frequent surveillance of expired tidal volume, respiratory rate, hemodynamics, and oxygenation is critical for timely escalation of support.

\section{Invasive mechanical ventilation}

While initial case series reported high mortality rates for patients receiving IMV for covid-19 pneumonia, ${ }^{34}$ these studies originated from hospitals that were overwhelmed with surges of covid-19 patients. Subsequent larger and complete series reported mortality rates consistent with classic ARDS when basic ARDS management tenets were followed. ${ }^{85137-141}$ In table 1 we summarize select large case series with detailed information on baseline characteristics, ventilator settings, and outcomes for patients receiving IMV.

Similar to HFNC and NIV, studies on IMV in the setting of covid-19 pneumonia suffer from retrospective design and lack of a control group. Notwithstanding, they indicate a striking resemblance in respiratory system mechanics and outcomes to classic ARDS.

The LUNG SAFE study reported the incidence, outcome, ventilator settings, adjunctive therapies, and outcomes of 2377 patients with classic ARDS who received IMV in 459 ICUs in 50 countries. ${ }^{142}$ The median age was 61 years, with nearly $60 \%$ of patients with pneumonia as the cause for ARDS. Patients remained on IMV for a median of 8 (4-16) days. Twenty eight day mortality was 35\% overall and $41 \%$ for those with severe ARDS. On presentation, median $\mathrm{P}_{\mathrm{a}} \mathrm{O}_{2} / \mathrm{F}_{\mathrm{i}} \mathrm{O}_{2}$ 161 (158-163) mm Hg, mean plateau pressure (Pplat) was $23.2(22.6-23.7) \mathrm{cm} \mathrm{H}_{2} \mathrm{O}$, delivered PEEP was 8.4 (8.3-8.6) $\mathrm{cm} \mathrm{H}_{2} \mathrm{O}$, and $\mathrm{FiO}_{2}$ 0.65. Adjunctive measures included $\mathrm{NMB}(22 \%)$, prone positioning $(8 \%)$, and ECMO (3\%).

Compared with observations in the LUNG SAFE study, IMV duration in CARDS case series may be slightly longer 85137138140 with higher rates of NMB use and prone positioning. ${ }^{85} 137140141$ Following the preliminary retrospective analysis of respiratory physiology during IMV, ${ }^{76}$ several larger prospective studies comparing consecutive typical ARDS and CARDS patients have been published. ${ }^{143-146}$ These studies essentially confirm the notion of similar respiratory mechanics and physiology between the two conditions; however with some interesting nuances. One study ${ }^{144}$ matched 30 CARDS patients with 30 typical ARDS patients based on oxygenation parameters, tidal volume, and PEEP. It confirmed similar respiratory system mechanics and demonstrated high recruitability $(\mathrm{R} / \mathrm{I}$ ratio $>0.5)$ in both CARDS (73\%) and ARDS (57\%) patients, in contrast to the preliminary analysis which showed low recruitability when supine. R/I ratio inversely correlated with $\mathrm{P}_{\mathrm{a}} \mathrm{CO}_{2}$ response to PEEP titration, suggesting hyperinflation and increase in dead space when recruitability was low. A study of 301 CARDS patients ${ }^{143}$ found similar respiratory system mechanics and lung weight as determined by computed tomography scan compared with a retrospective cohort of typical ARDS patients. The investigators identified that those with a lower respiratory system compliance $\left(<41 \mathrm{~mL} / \mathrm{cm} \mathrm{H}_{2} \mathrm{O}\right)$ and high D-dimer had higher mortality compared with other subgroups. Ventilatory ratio (the product of tidal volume, ventilatory rate, and $\mathrm{PaCO}_{2}$, indexed for predicted body weight), which is a marker for dead space, also correlated with D-dimer levels raising suspicion for pulmonary intravascular thrombosis.

A contentious issue in IMV is when to intubate patients with CARDS. Two retrospective cohort studies of covid-19 patients have reported different conclusions, with one favoring earlier intubation ${ }^{147}$ and the other finding no association of mortality with time to intubation or HFNC use. ${ }^{148}$ Intensivists have struggled with this dilemma since the beginning of mechanical ventilation ${ }^{149}$ : triggers for initiating IMV in clinical studies and in practice are not standardized and may depend on various factors including clinical judgment, severity of illness, patient preference, and cultural norms regarding mechanical ventilation. In the case of covid-19 pneumonia, resource limitation, hypothetical concerns over P-SILI, ${ }^{83}$ and expert opinion on NIV may have played a role in the adoption of early IMV. Given the favorable outcomes of HFNC trials in classic ARDS, ${ }^{8990}$ we speculate that the likelihood of harm is small when standardized indices for detecting respiratory failure are applied and patients are transitioned to IMV when clinically indicated.

\section{Tracheobronchial hygiene}

Patients on mechanical ventilation for covid-19 pneumonia may develop increased mucus production with airflow obstruction. In a large cohort of covid-19 patients who underwent tracheostomy, most of the endotracheal tubes were partially occluded with sticky secretions. ${ }^{150}$ This manifestation may be due to changes in mucus regulation caused by SARS-CoV2 infection. ${ }^{151}$ Effective humidification, monitoring airway resistance, and potentially the use of mucolytics and endotracheal tube clearing devices ${ }^{152}$ may be helpful.

\section{Weaning and tracheostomy}

We found no pertinent studies evaluating strategies for weaning from mechanical ventilation for covid-19 


\section{Table 1 | Studies on respiratory assist devices in covid-19 (HFNC, NIV, and IMV)}

\begin{tabular}{|c|c|c|c|c|c|c|c|}
\hline Study & N (HFNC) & $\begin{array}{l}\text { Age median } \\
\text { (interquartile } \\
\text { range) }\end{array}$ & $\begin{array}{l}\text { Gender } \\
(\% \mathrm{~F})\end{array}$ & $\begin{array}{l}\text { Oxygenation at } \\
\text { baseline } \mathrm{PaO}_{2} / \mathrm{Fi}_{0} 2\end{array}$ & Duration of use & Outcome & Comment \\
\hline \multicolumn{8}{|c|}{ Selected studies on high flow nasal cannula } \\
\hline Calligaro et al & 293 & $52(44-58)$ & 44 & $68(54-92)$ & $\begin{array}{l}6(3-9) \text { days-success } \\
2(1-5) \text { days failure }\end{array}$ & $\begin{array}{l}47 \% \text { success on HFNC alone. } \\
\text { Overall survival to discharge } \\
52 \%\end{array}$ & $\begin{array}{l}\text { ROX-6 } h \geq 3.7,80 \% \text { success } \\
\text { ROX-6 } h \leq 2.2,74 \% \text { failure }\end{array}$ \\
\hline Ferrando et al & 199 & $\begin{array}{l}\text { HFNC } \\
\text { only: } \\
63(55-71) \\
\text { HFNC+ } \\
\text { Prone: } \\
60(54-70)\end{array}$ & 26 & $\begin{array}{l}\text { HFNC only } 111(83-144) \\
\text { HFNC+prone } 125 \text { (99- } \\
\text { 187) }\end{array}$ & $\begin{array}{l}\text { Treatment duration } \\
\text { not available } \\
\text { ICU length of stay } \\
7.5-8 \text { days }\end{array}$ & $\begin{array}{l}\text { HFNC success } 58.3 \% \\
\text { +prone } 60 \% \\
\text { ICU mortality } 13.9 \% \text { and } \\
16.3 \% \text { respectively }\end{array}$ & $\begin{array}{l}\text { No difference in outcomes when } \\
\text { proning HFNC patients. Potential } \\
\text { delay in intubation when proning }\end{array}$ \\
\hline $\begin{array}{l}\text { Demoule A } \\
\text { et al }\end{array}$ & 146 & $60(53-67)$ & 21 & $126(86-189)$ & $4(2-6)$ & 28 day mortality $21 \%$ & $\begin{array}{l}\text { HFNC reduced intubation rate } \\
\text { without affecting case fatality } \\
\text { (propensity score matched } \\
\text { analysis versus those who did } \\
\text { not receive HFNC) }\end{array}$ \\
\hline Zucman et al & 62 & $55(48-63)$ & $\begin{array}{l}\text { Not } \\
\text { reported }\end{array}$ & $\begin{array}{l}\mathrm{F}_{1} \mathrm{O}_{2} 0.8(0.6-1) \\
\mathrm{SpO}_{2} 96(94-98) \%\end{array}$ & $\begin{array}{l}10 \text { hours }(7-57) \text { for } \\
\text { failure }\end{array}$ & $\begin{array}{l}34 \% \text { success on HFNC alone. } \\
\text { Overall ICU mortality } 17 \%\end{array}$ & $\begin{array}{l}\text { ROX-4 } h \geq 5.37 \text {, lower risk } \\
\text { of intubation HR } 0.59 \text { ( } 95 \% \\
\text { confidence interval } 0.41 \text { to } 0.84 \text { ) }\end{array}$ \\
\hline Xia et al & 43 & 64 SD 9.7 & 42 & $122.3 \pm 51.3 \mathrm{~mm} \mathrm{Hg}$ & $\begin{array}{l}4(2-7) \text { days } \\
5 \text { (3-7) days } \\
\text { success } \\
3.5(1.5-6.5) \\
\text { days failure }\end{array}$ & $\begin{array}{l}53.5 \% \text { success on HFNC } \\
\text { alone. } \\
\text { Hospital mortality } 32.5 \% \text { for } \\
\text { entire cohort ( } 65 \% \text { if HFNC } \\
\text { failure) }\end{array}$ & $\begin{array}{l}\text { Male sex and lower oxygenation } \\
\text { on admission risk factors for } \\
\text { failure. Overall mortality } 32.5 \% \text {. } \\
\text { Mortality } 65 \% \text { for invasive } \\
\text { mechanical ventilation }\end{array}$ \\
\hline Panadero et al & 40 & 58.9 SD 11.8 & $30 \%$ & $\begin{array}{l}\mathrm{S}_{\mathrm{p}} \mathrm{O}_{2} / \mathrm{F}_{\mathrm{i}} \mathrm{O}_{2} \\
113.4 \pm 6.6 \text { (success) } \\
93.7 \pm 6.7 \text { (failure) }\end{array}$ & $\begin{array}{l}6(5-8) \text { days success } \\
2(1-4) \text { days failure }\end{array}$ & $\begin{array}{l}\text { Not intubated at } 30 \text { days } \\
47.5 \% \\
\text { Mortality } 22.5 \%\end{array}$ & $\begin{array}{l}\text { ROX } 2-6<4.94 \text { associated with } \\
\text { high risk of intubation HR } 4.03 \\
(1.18-13.7)\end{array}$ \\
\hline Vianello et al & 28 & $69(42-87)$ & 33.3 & $108(52-296)$ & Not available & $\begin{array}{l}67.8 \% \text { discharged alive. } \\
17.8 \% \text { required IMV } \\
15 \text { day mortality } 11 \% \text { (3 } \\
\text { patients on IMV) }\end{array}$ & $\begin{array}{l}\text { Patients with } \mathrm{P}_{a} \mathrm{O}_{2} / \mathrm{F}_{i} \mathrm{O}_{2} \leq 100 \text { had } \\
\text { failure rate of } 77.8 \% \text {. } \\
\text { Among the } 73 \text { healthcare } \\
\text { workers who took care of the } \\
\text { patients for an average of } 48 \\
\text { hours per person, no infections } \\
\text { were reported }\end{array}$ \\
\hline Guy et al & 27 & $77(77-79)$ & 19 & $124(120-158)$ & $6(2-10)$ days & $\begin{array}{l}70 \% \text { HFNC success. } \\
26 \% \text { required invasive } \\
\text { mechanical ventilation. } \\
\text { Overall mortality } 15 \%\end{array}$ & $\begin{array}{l}\text { Consecutive patients treated in a } \\
\text { non-ICU setting }\end{array}$ \\
\hline Duan et al & 23 & 65 SD14 & $48 \%$ & $196 \pm 46$ & $\begin{array}{l}3.6 \text { days } \\
(1.6-8.4)\end{array}$ & $\begin{array}{l}57 \% \text { HFNC success. } \\
43 \% \text { transitioned to NIV } \\
17 \% \text { eventually intubated } \\
\text { mortality } 4 \%\end{array}$ & $\begin{array}{l}\text { Elevated } \mathrm{C} \text { reactive protein } \\
\text { predicted intubation }\end{array}$ \\
\hline Wang et al & 17 & $65(56-75)$ & 59 & 209 (179-376) & 76 hours & $59 \%$ HFNC success & $\begin{array}{l}\text { If } \mathrm{P}_{\mathrm{a}} \mathrm{O}_{2} / \mathrm{F}_{\mathrm{i}} \mathrm{O}_{2}>200,0 \% \text { failure. } \\
\text { Reduction in respiratory rate after } \\
1-2 \mathrm{~h} \text { on HFNC predicted success }\end{array}$ \\
\hline \multicolumn{8}{|c|}{ Studies on non-invasive ventilation } \\
\hline Bellani G et al & 798 & $68(59-75$ & 26 & $168(98)$ & Cross sectional study & $\begin{array}{l}62.4 \% \text { were discharged alive } \\
\text { without needing intubation }\end{array}$ & $\begin{array}{l}\text { NIV outside of ICU } \\
68 \% \text { were treated with helmet } \\
\text { CPAP. } \\
53 \% \text { NIV failure when P/F ratio } \\
<150 \mathrm{~mm} \mathrm{Hg}\end{array}$ \\
\hline Aliberti et al & 157 & $64(55-75)$ & 25.5 & $142.9(96.7-203.2)$ & $\begin{array}{l}\text { CPAP success } 8 \\
(5-14) \\
\text { CPAP failure } \\
4(3-7)\end{array}$ & $\begin{array}{l}\text { Success } 55.4 \% \text {. Hospital } \\
\text { mortality } 28.7 \%\end{array}$ & $\begin{array}{l}\text { Helmet CPAP in a respiratory unit } \\
\text { (high dependency unit) } \\
41.4 \% \text { of patients DNI } \\
\text { Severe pneumonia, elevated IL- } 6 \\
\text { associated with failure. }\end{array}$ \\
\hline Hua et al & 152 & 67 SD 13 & 46 & Not provided & $\begin{array}{l}\text { Length of stay } 16.1 \\
\pm 9.6 \text { days }\end{array}$ & Survival $59.2 \%$ & $\begin{array}{l}\text { Higher incidence of COPD in NIV } \\
\text { patients }\end{array}$ \\
\hline Wang et al & $\begin{array}{l}122 \\
\text { (Full cohort } \\
141)\end{array}$ & $\begin{array}{l}64(55-70) \\
\text { (Includes full } \\
\text { cohort) }\end{array}$ & 30 & $\begin{array}{l}\text { NIV } 261.9(218.6- \\
314.3) \\
\text { NIV+IMV } \\
233.3(118-278.6)\end{array}$ & Not reported & $\begin{array}{l}75 \% \text { success. Mortality } 17 \% \\
\text { (incomplete data) }\end{array}$ & $\begin{array}{l}\text { D-dimer }>1.5 \mathrm{mg} / \mathrm{L} \text { increased } \\
\text { likelihood of IMV OR } 3.28 \\
(1.07-10.1)\end{array}$ \\
\hline Duca et al & $\begin{array}{l}\text { Helmet } \\
\text { CPAP } 71 \\
\text { BiPAP } 7\end{array}$ & $70(62-79)$ & 16 & $\begin{array}{l}131(97-190) \\
87(53-120)\end{array}$ & Not reported & $\begin{array}{l}\text { CPAP } \\
14 \% \text { survival } \\
\text { BiPAP } 42.9 \% \text { survival }\end{array}$ & $\begin{array}{l}54.9 \% \text { of helmet CPAP patients } \\
\text { died before intubation. }\end{array}$ \\
\hline $\begin{array}{l}\text { Sivaloganathan } \\
\text { et al }\end{array}$ & $\begin{array}{l}58 \\
\text { (NIV } \pm \text { IMV) } \\
24 \text { (NIV } \\
\text { only) }\end{array}$ & $\begin{array}{l}\text { NIV success } 50 \\
(45-60) \\
\text { NIV failure } \\
57(50-64) \\
\text { NIV only } \\
66(54-72)\end{array}$ & 43 & Not provided & $\begin{array}{l}17 \text { hours }(4-31) \\
\text { failure } \\
72 \text { hours (41-132) } \\
\text { Success }\end{array}$ & $\begin{array}{l}\text { Success } \\
53 \% \text { (31/58 pts). } \\
\text { NIV+IMV group } 11.1 \% \\
\text { mortality (incomplete } \\
\text { outcome). } \\
\text { NIV ceiling (DNI) group } \\
\text { mortality } 83.3 \%\end{array}$ & $\begin{array}{l}\text { Admission SOFA score predicted } \\
\text { risk of intubation }\end{array}$ \\
\hline
\end{tabular}


Table 1 | Studies on respiratory assist devices in covid-19 (HFNC, NIV, and IMV)

\begin{tabular}{|c|c|c|c|c|c|c|c|}
\hline Study & $\mathrm{N}$ (HFNC) & $\begin{array}{l}\text { Age median } \\
\text { (interquartile } \\
\text { range) }\end{array}$ & $\begin{array}{l}\text { Gender } \\
(\% \mathrm{~F})\end{array}$ & $\begin{array}{l}\text { Oxygenation at } \\
\text { baseline } \mathrm{PaO}_{2} / \mathrm{Fi}_{0} 2\end{array}$ & Duration of use & Outcome & Comment \\
\hline Oranger et al & $\begin{array}{l}38 \\
\text { (All CPAP) } \\
14 \\
\text { controls } \\
\text { (no CPAP) }\end{array}$ & $63(55-70)$ & 32 & $\begin{array}{l}\mathrm{P}_{\mathrm{a}} \mathrm{O}_{2} 71(63.5-88.5) \\
\mathrm{On} 5(3-6) \mathrm{L} / \mathrm{min} \mathrm{O} 2\end{array}$ & 5 days & $\begin{array}{l}\text { Success } 77 \% \text { v } 43 \% \text { in } \\
\text { controls }\end{array}$ & $\begin{array}{l}\text { CPAP applied in the ward. } \\
\text { None of the intubations were } \\
\text { emergent }\end{array}$ \\
\hline Burns et al & 28 & $81.5(54-91)$ & 46 & Not provided & 5 days $(1-14)$ & $\begin{array}{l}50 \% \text { survival to discharge } \\
\text { CPAP mortality } 52 \% \text {, BiPAP } \\
\text { mortality } 40 \%\end{array}$ & $\begin{array}{l}23 / 28 \text { received } \mathrm{CPAP} \\
\text { (Average } 13 \mathrm{~cm} \mathrm{H}_{2} \mathrm{O} \text { ) } \\
\text { Ventilation provided in the ward }\end{array}$ \\
\hline Zheng et al & 19 & $66(51-72)$ & 27 & Not available & Not available & $95 \%$ discharged & $\begin{array}{l}\text { Thrombocytopenia and high } \\
\text { IL-6 levels more common in IMV } \\
\text { compared with NIV }\end{array}$ \\
\hline Duan et al & 13 & 50 SD 14 & 8 & $165 \pm 48$ & 7 days & $\begin{array}{l}85 \% \text { success with NIV } \\
\text { Mortality } 8 \%\end{array}$ & $\begin{array}{l}\text { NIV and HFNC first strategy had } \\
\text { comparable outcomes }\end{array}$ \\
\hline \multicolumn{8}{|c|}{ Studies on invasive mechanical ventilation } \\
\hline $\begin{array}{l}\text { Graselli et al } \\
\text { Lombardy, Italy }\end{array}$ & $\begin{array}{l}1150 \\
\text { (full cohort } \\
1591 \text { ) }\end{array}$ & $63(56-70)$ & 18 & $\begin{array}{l}\mathrm{P}_{\mathrm{a}} \mathrm{O}_{2} / \mathrm{F}_{\mathrm{i}} \mathrm{O}_{2} \\
160(114-220) \mathrm{mm} \mathrm{Hg} \\
\text { No lung mechanics } \\
\mathrm{PEEP} 14(12-16) \mathrm{cm} \\
\mathrm{H}_{2} \mathrm{O} \\
\mathrm{FiO} 20.7(0.5-0.8 \\
\end{array}$ & $\begin{array}{l}\text { ICU length of stay } 9 \\
(6-13) \text { days }\end{array}$ & $\begin{array}{l}\text { ICU mortality } 26 \% \\
920 / 1591 \text { still in the ICU }\end{array}$ & $\begin{array}{l}27 \% \text { were prone } \\
\text { No data on NMB } \\
\text { ECMO } 1 \%\end{array}$ \\
\hline $\begin{array}{l}\text { Ferrando et al } \\
36 \text { Spanish } \\
\text { Andorran ICUs }\end{array}$ & 742 & $64(56-71)$ & 31.9 & $\begin{array}{l}\mathrm{P}_{2} \mathrm{O}_{2} / \mathrm{F}_{1} \mathrm{O}_{2} \\
120(83-177) \mathrm{mm} \mathrm{Hg} \\
\text { Static compliance: } 35 \\
\text { (27-45) } \mathrm{ml} / \mathrm{cm}_{2} \mathrm{O} \\
\text { Plateau pressure } 25(22- \\
29) \mathrm{cm}_{2} \mathrm{O} \\
\text { Driving pressure } 12 \mathrm{~cm} \\
\mathrm{H}_{2} \mathrm{O}(10-16)\end{array}$ & $\begin{array}{l}\text { Ventilator length of } \\
\text { stay } 14 \text { (7-24) days }\end{array}$ & 28 day mortality $32 \%$ & $\begin{array}{l}\text { Most common comorbidities HTN } \\
\text { and obesity. } \\
<10 \% \text { of cohort still in ICU } \\
\text { Static compliance not related to } \\
\text { outcome } \\
\text { Lung protective ventilation, } \\
\text { NMB }(72 \%) \text {, proning }(76 \%) \text {, } \\
\text { recruitment maneuvers(79\%) } \\
\text { common }\end{array}$ \\
\hline $\begin{array}{l}\text { Schenck et al } \\
\text { NY } \\
\text { Presbyterian } \\
\text { Hospital- } \\
\text { Weill Cornell } \\
\text { Medicine }\end{array}$ & 267 & $66(54-74)$ & 28 & $\begin{array}{l}\mathrm{P}_{2} \mathrm{O}_{2} / \mathrm{F}_{\mathrm{O}_{2}} \\
103(82-134) \mathrm{mm} \mathrm{Hg} \\
\text { Static compliance } 28 \\
\text { (23-38) } \mathrm{ml} / \mathrm{cm} \mathrm{H}_{2} \mathrm{O} \\
\text { Plateau pressure } 25 \\
(21-29) \mathrm{Cm} \mathrm{H}_{2} \mathrm{O} \\
\text { Driving pressure } 14 \\
\text { (11-17.2) } \mathrm{cm} \mathrm{H}_{2} \mathrm{O}\end{array}$ & $\begin{array}{l}\text { Currently intubated } \\
18(14-24) \\
\text { Extubated } 10(6-15) \\
\text { Deceased } \\
8(4-13)\end{array}$ & $\begin{array}{l}\text { More than half of the cohort } \\
\text { remained intubated (141 } \\
\text { patients) } \\
49 \text { patients died (18.4\%) } \\
77 / 267 \text { extubated } \\
49 / 267 \text { Deceased } \\
141 / 267 \text { Intubated }\end{array}$ & $\begin{array}{l}\text { Longer intubation periods } \\
\text { compared to typical ARDS } \\
\text { NMB } 60 \% \\
\text { Prone } 40 \% \\
25 \% \text { had static compliance }>38 \\
\mathrm{~mL} / \mathrm{cm} \mathrm{H}_{2} \mathrm{O}\end{array}$ \\
\hline $\begin{array}{l}\text { Cummings et al } \\
2 \text { NY } \\
\text { Presbyterian } \\
\text { hospitals } \\
\text { (Columbia } \\
\text { Univ.) }\end{array}$ & $\begin{array}{l}203 \\
\text { (Full cohort } \\
257)\end{array}$ & $62(51-72)$ & 33 & $\begin{array}{l}\mathrm{P}_{\mathrm{a}} \mathrm{O}_{2} / \mathrm{F}_{\mathrm{i}} \mathrm{O}_{2} \\
129(80-203) \mathrm{mm} \mathrm{Hg} \\
\text { Driving pressure } 15 \\
(11-18) \mathrm{Cm} \mathrm{H}_{2} \mathrm{O} \\
\mathrm{PEEP} 15(12-18) \mathrm{cm} \\
\mathrm{H}_{2} \mathrm{O} \\
\mathrm{FiO}^{2} 1(0.8-1) \\
\mathrm{Plateau} \text { pressure } 27 \\
(23-31) \mathrm{Cm}_{2} \mathrm{O}\end{array}$ & $18(9-28)$ days & $\begin{array}{l}\text { In-hospital mortality } 39 \% \\
23 \% \text { discharged alive } \\
2 \% \text { transfer to another } \\
\text { hospital } \\
37 \% \text { remained hospitalized }\end{array}$ & $\begin{array}{l}25 \% \text { received NMB } \\
17 \% \text { proned } \\
\text { ECMO } 3 \%\end{array}$ \\
\hline $\begin{array}{l}\text { Auld et al } \\
\text { Emory } \\
\text { Healthcare } \\
\text { acute care } \\
\text { hospitals }\end{array}$ & $\begin{array}{l}165 \\
\text { (Full cohort } \\
217 \text { ) }\end{array}$ & $64(54-73)$ & 45.2 & $\begin{array}{l}\mathrm{P}_{\mathrm{a}} \mathrm{O}_{2} / \mathrm{F}_{1} \mathrm{O}_{2} \\
132(100-178) \mathrm{mm} \mathrm{Hg} \\
\text { Static lung compliance } \\
34(28-46) \mathrm{mL} / \mathrm{cm} \mathrm{H}_{2} \mathrm{O}\end{array}$ & $\begin{array}{l}\text { Ventilator days } \\
9(4-13)\end{array}$ & $\begin{array}{l}\text { Mortality for ventilated } \\
\text { patients } \\
33.9 \%\end{array}$ & $\begin{array}{l}\text { Institutional adoption of early } \\
\text { intubation and lung protective } \\
\text { strategy. } \\
\text { NMB or proning data not } \\
\text { available } \\
\text { ECMO } 1.8 \%\end{array}$ \\
\hline $\begin{array}{l}\text { Ziehr et al } \\
\text { MA. General } \\
\text { Hospital and } \\
\text { Beth Israel } \\
\text { Deaconess } \\
\text { Medical Center }\end{array}$ & 66 & $58(23-87)$ & 35 & $\begin{array}{l}\mathrm{P}_{\mathrm{a}} \mathrm{O}_{2} / \mathrm{F}_{1} \mathrm{O}_{2} \\
182(135-245) \mathrm{mm} \mathrm{Hg} \\
\text { Static compliance } 35 \\
\text { (30-43) } \mathrm{mL} / \mathrm{cm} \mathrm{H}_{2} \mathrm{O} \\
\text { Driving pressure } 11 \\
(9-12) \mathrm{cm} \mathrm{H}_{2} \mathrm{O}\end{array}$ & $16(10-21)$ days & $\begin{array}{l}\text { Mortality } 16.7 \% \\
62 \% \text { extubated } \\
21.2 \% \text { received tracheostomy } \\
\text { Length of stay } 17.5(13-25)\end{array}$ & $\begin{array}{l}42 \% \text { received NMB } \\
47 \% \text { proned } \\
95 \% \text { on vasopressors } \\
5 \% \text { received ECMO }\end{array}$ \\
\hline $\begin{array}{l}\text { Bhatraju et al } \\
9 \text { Seattle } \\
\text { hospitals }\end{array}$ & $\begin{array}{l}18 \\
24 \\
\text { patients in } \\
\text { cohort }\end{array}$ & 64 SD 8 & 37 & $\begin{array}{l}\mathrm{P}_{\mathrm{a}} \mathrm{O}_{2} / \mathrm{F}_{1} \mathrm{O}_{2} \\
142(94-177) \mathrm{mm} \mathrm{Hg} \\
\text { Compliance } 29(25-36) \\
\mathrm{mL} / \mathrm{cm} \mathrm{H}_{2} \mathrm{O} \\
\text { Driving pressure } 13 \\
(11-17) \mathrm{cm} \mathrm{H}_{2} \mathrm{O} \\
\text { Plateau pressure } 25 \\
(20-28) \mathrm{Cm}_{2} \mathrm{O} \\
\mathrm{FiO} 20.9(0.7-1)\end{array}$ & $10(7-12)$ days & $\begin{array}{l}33 \% \text { extubated } \\
50 \% \text { mortality } \\
17 \% \text { still on mechanical } \\
\text { ventilator }\end{array}$ & $\begin{array}{l}\text { NMB } 39 \% \\
\text { Prone } 28 \% \\
71 \% \text { on vasopressors } \\
\text { Older age associated with poor } \\
\text { outcomes }\end{array}$ \\
\hline
\end{tabular}


patients. Some authors recommend heightened caution because of the risk to healthcare workers during the process of extubation and reintubation following weaning failure. ${ }^{153}$ Novel procedures such as the "mask over tube" extubation can potentially reduce exposure to droplets and aerosols. ${ }^{154}$ In the absence of evidence to the contrary, we recommend no changes to the established stages to weaning from mechanical ventilation. ${ }^{155}$ Extubation can be safely performed while adhering to standard PPE practices.

Tracheostomy may be necessary in approximately $13 \%$ of typical ARDS patients to facilitate continued weaning. ${ }^{156}$ However, tracheostomy is considered an aerosol generating procedure. During the SARS epidemic, those involved in performing tracheostomy had $>4$ higher odds of contracting disease. ${ }^{157}$ Hesitation to perform the procedure during the early days of the pandemic was justifiable, therefore. Several large series since then show favorable outcomes and safetyfor tracheostomyinmanaging covid-19. ${ }^{150158159}$ In a national cohort study from Spain, ${ }^{150} 1890$ tracheostomies were performed within seven weeks for critically ill covid-19 patients. The investigators reported a median of 12 (4-42) days from intubation to the procedure. More than half of the patients were weaned (52\%) and mortality was 24\%. Open tracheostomies were preferred over percutaneous approach $(81.3 \%$ versus $18.7 \%)$. No disease transmission incidents were reported among the staff performing the studies. ${ }^{150} 158159$ In one study, early tracheostomy (<10 days from intubation) was associated with shorter IMV duration (mean (SD), 18 (5.4) $v 22.3$ (5.7) days). ${ }^{159}$ The type of surgical technique (percutaneous versus open) and timing of tracheostomy were not associated with complications or mortality. ${ }^{158}$ Several multidisciplinary guidelines have been put together to ensure optimal outcomes and safety. ${ }^{160}{ }^{161}$ Tracheostomy appears feasible and safe among covid-19 patients and could facilitate earlier weaning and enhance availability of mechanical ventilators.

\section{Covid-19 drug treatments}

From a mechanistic perspective, treatments targeting viral replication could be more effective early in the disease process (eg, antiviral therapies like remdesivir, passive antibody therapies like monoclonal antibodies, and convalescent plasma). Later in the disease course, when an excess and inappropriate immune response is responsible for pathology and illness, anti-inflammatory treatments like corticosteroids could be more effective. It is important for clinicians to diagnostically classify the clinical presentation of the patient by severity of clinical disease, and consider whether a patient has mild/moderate disease (not requiring supplemental oxygen), severe (requiring low flow oxygen), or critical covid-19 (on HFNC, NIV, IMV, or ECMO) which has major implications for the choice of pharmacologic treatment and management. We have summarized the recommended treatments in table 2. Treatment with monoclonal antibodies is currently not recommended for patients hospitalized for covid-19 and is not within the scope of our review. ${ }^{162} 163$

\section{Corticosteroids}

Corticosteroids are the only therapeutic agents that have demonstrated a clear mortality benefit in the treatment of severe covid-19. Seven RCTs have evaluated treatment with steroids in critically ill patients ${ }^{164}$ and one trial in severe non-critical covid-19, ${ }^{165}$ including medium and high dose dexamethasone, hydrocortisone, and methylprednisolone. In the largest trial $(n=2104)$, 28 day mortality was $22.9 \%$ in the dexamethasone arm compared with $25.7 \%$ in usual care (adjusted rate ratio 0.83 , confidence interval 0.75 to 0.93 ). The patients with the highest mortality reduction were those on IMV compared with usual care (dexamethasone 29.3\% versus usual care 41.4\%; rate ratio 0.64 , confidence interval 0.51 to 0.81 ). Those needing supplemental oxygen also had a mortality reduction but the effect size was smaller (dexamethasone 23.3\% versus usual care $26.2 \%$; rate ratio 0.82 ; confidence interval 0.72 to 0.94$)$. Patients mild to moderately ill and not on supplemental oxygen had a non-significant increase in mortality rate (dexamethasone $17.8 \%$ versus usual care $14.0 \%$; rate ratio 1.19 , confidence interval 0.91 to 1.55$)$. A meta-analysis that pooled data from all the RCTs of steroids showed a significant decrease in mortality for dexamethasone (fixed effect odds ratio 0.64 , confidence interval 0.50 to 0.82 for dexamethasone from three trials, $n=1282$ ) and a non-significant decrease for hydrocortisone (odds ratio 0.69 , confidence interval 0.43 to $1.12 ; \mathrm{P}=0.13$, $\mathrm{n}=374$ ). No significant mortality reduction was seen with methylprednisolone but this was based on one trial with 47 patients (odds ratio 0.91, confidence interval 0.29 to $2.87 ; \mathrm{P}=0.87) .{ }^{165}$ We believe that, while the evidence is most robust for dexamethasone and hydrocortisone, no evidence exists at present to believe one steroid is superior to the other. Head-tohead studies comparing the different types of steroid are needed.

\section{Remdesivir}

Remdesivir is an antiviral drug that acts by inhibiting viral RNA transcription. ${ }^{166}$ It has in vitro activity against many RNA viruses including SARS CoV2. Current studies have been done in hospitalized patients with moderate or severe disease.

\section{Remdesivir for moderate covid-19}

SIMPLE- $2^{160}$ was an RCT specifically designed to evaluate remdesivir in hospitalized patients with moderate covid-19 (not needing supplemental oxygen), although ACCT- $1^{167}$ and SOLIDARITY ${ }^{168}$ also included patients with moderate disease. SIMPLE-2 compared a course of five to 10 days of redemsivir with standard care. The 5 day group had higher odds (odds ratio 1.65; 95\% confidence interval 1.09 to 2.48; $\mathrm{P}=0.02$ ) for improved clinical status using a 


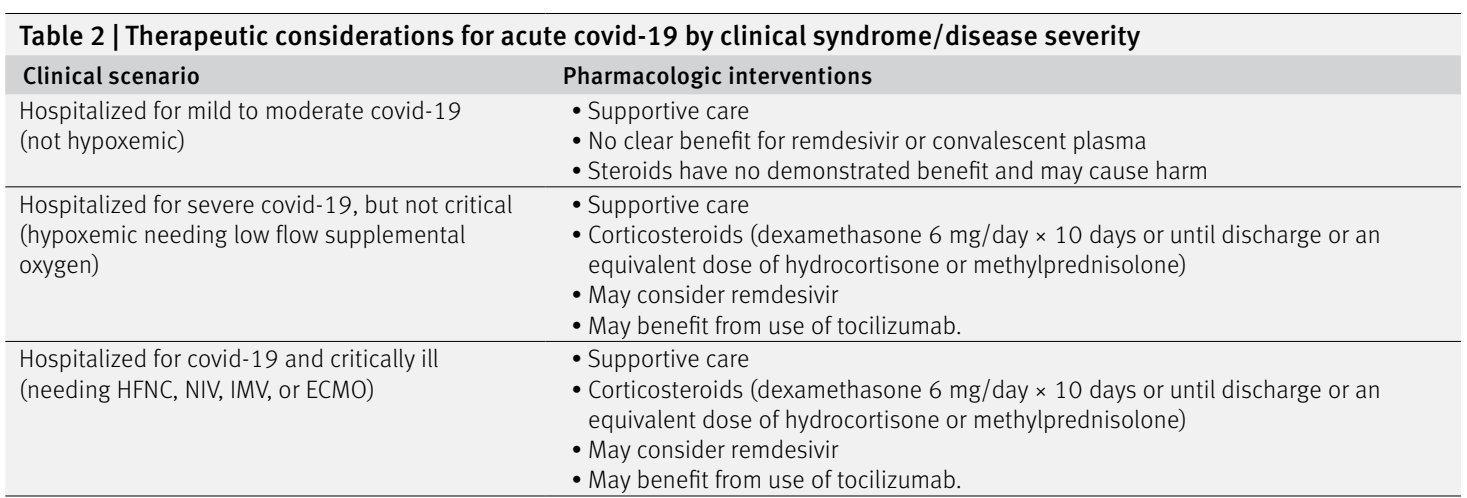

composite severity of illness score (eg,1=discharge from hospital, 7 =death). No statistically significant difference was seen between clinical status on day 11 with the 10 day course of remdesivir to standard care $(\mathrm{P}=0.18$ by Wilcoxon rank sum test), and no significant difference in outcomes such as time to recovery, duration of treatment with supplemental oxygen, duration of hospitalization, or mortality. ${ }^{169}$ Results from the other two studies that included patients with moderate covid-19 also did not show a mortality benefit.

\section{Remdesivir for severe covid-19}

Three RCTs (SIMPLE-1, ACCT-1, and SOLIDARITY) evaluated remdesivir in hospitalized patients with severe covid-19 (oxygen saturation $<94 \%$ on room air requiring supplemental oxygen or more advanced respiratory support/ECMO). ${ }^{167168170}$ ACCT1 showed earlier time to recovery and discharge from remdesivir, but no mortality benefit compared with placebo (median 10 days with remdesivir compared with 15 days with placebo; rate ratio for recovery 1.29; confidence interval 1.12 to 1.49 ). A post hoc sub-analysis showed the largest effect size for recovery was in patients requiring low flow oxygen who were not critically ill $(n=957$, median time to recovery 11 versus 18 days, rate ratio for recovery 1.31; confidence interval 1.12 to 1.52 ). The rate ratios for recovery in those critically ill (need for HFNC, NIV, IMV, or ECMO) were not statistically significant compared with placebo. Given the smaller number of patients in these subgroups it is unclear if this difference is due to an inadequate sample size or if remdesivir was not effective. Also, some of the outcomes used to create the 7-point ordinal scale for clinical improvement could have been influenced by resource limitations (ie, ventilator availability) or regional practices. SOLIDARITY $(n=2700)$, the largest trial to date, showed that remdesivir was not associated with a reduction in mortality or rates of IMV (mortality rate ratio 0.95, confidence interval 0.81 to $1.11, \mathrm{P}=0.50 ; 301 / 2743$ remdesivir versus $303 / 2708$ control). Despite the limitation that this was an open label study with no placebo, the outcomes for mortality or need for IMV are less prone to bias than subjective clinical outcomes. The third study (SIMPLE-1) ${ }^{171}$ compared five to 10 days of treatment in hospitalized patients with severe non-critical disease. The 5 day course showed better clinical improvement at day 14, but patients in the 10 day arm had more severe disease raising the concern for confounding even after adjustment.

In summary, remdesivir may have modest benefit in time to recovery in patients with severe disease, but shows no significant benefit in mortality or other clinical outcomes.

\section{Tocilizumab}

Tocilizumab is a monoclonal antibody that blocks the IL- 6 receptor and is used to treat cytokine release syndrome associated with CAR-T cell therapy. Multiple case series and observational studies were published in the early months of the pandemic that reported improved outcomes from tocilizumab. ${ }^{172-174}$ Since then, eight RCTs have compared tocilizumab with placebo or standard care in severe covid-19. ${ }^{175-182}$ Some of the largest trials have only preprints available (COVATA ${ }^{176}$, REMAPCAP ${ }^{180}$ and RECOVERY). ${ }^{182}$ EMPACTA was conducted in hospitalized non-ventilated patients with covid-19 and included high risk racial and ethnic minority patients. While this RCT reported a benefit for the composite outcome of mortality and need for IMV in the tocilizumab arm, it did not show mortality benefit alone. The cumulative proportion of IMV or mortality on day 28 for tocilizumab was $12.0 \%$ versus placebo 19.3\% ( $\log$ rank $\mathrm{P}=0.0360$; hazard ratio 0.56 ; confidence interval 0.33 to 0.97 , and all cause mortality at day 28 for tocilizumab was $10.4 \%$ versus $8.6 \%$ (weighted difference $2.0 \%$, confidence interval -5.2 to 7.8). COVACTA included patients with severe illness and critical patients and reported no differences in mortality (19.7\% versus $19.4 \%$ in the placebo group at day 28 ; difference $0.3 \%$, confidence interval -7.6 to 8.2) or when utilizing an ordinal scale for clinical improvement (odds ratio 1.19, confidence interval 0.81 to 1.76 ).

REMAP-CAP was a randomized adaptive platform open label trial ( $n=353$ tocilizumab, $n=402$ usual care). Tocilizumab was administered within 24 hours of being admitted to an ICU and most also received corticosteroids. The median organ support-free days were 10 (IQR -1, 16), and 0 (IQR -1, 15) for tocilizumab and control, respectively. Hospital mortality was $28 \%$ (98/350) for tocilizumab and 35.8\% (142/397) for control. The authors used bayesian statistics and 


\begin{tabular}{|c|c|}
\hline System & Complications \\
\hline Physical impairment & $\begin{array}{l}\text { - Seen in up to } 80 \% \text { after any critical illness and includes loss of muscle mass, neuromuscular weakness, fatigue, dyspnea, decreased exercise } \\
\text { tolerance, joint contractures, and sexual dysfunction. }{ }^{190-192} \\
\text { - Substantial muscle wasting and neuromuscular weakness are common following non-covid ARDS and can last for months or years, }{ }^{193} \text { with major } \\
\text { risk factors being corticosteroid use and intensive care unit length of stay }{ }^{194} \\
\text { - Recent study from Italy of covid-19 patients with more than half reporting 3+ persistent symptoms, including fatigue (53\%), dyspnea ( } 43 \%) \text {, joint } \\
\text { pain ( } 27 \%) \text {, and reductions in quality of life }(44 \%)^{195}\end{array}$ \\
\hline $\begin{array}{l}\text { Mental health } \\
\text { impairment }\end{array}$ & $\begin{array}{l}\text { - For non-covid patients who were in intensive care unit, these include anxiety, depression, or post-traumatic stress disorder (PTSD) in } 8 \% \text { to } 57 \% \text { of } \\
\text { cases }{ }^{196-198} \\
\text { - Can also occur in family members of patients who were in intensive care units (known as PICS-family) } \\
\text { - Unique to covid-19 which increase the risk for mental health impairment include social isolation, loneliness, the stigma of the disease, limited } \\
\text { hospital visitation policy, and the psychological effect of the pandemic itself }{ }^{199} \\
\text { - In a study of } 402 \text { survivors of covid-19, a significant number of patients reported PTSD (28\%), depression (31\%), anxiety (42\%), obsessive- } \\
\text { compulsive symptomatology ( } 20 \%) \text {, and insomnia ( } 40 \%)^{200}\end{array}$ \\
\hline Pulmonary impairment & $\begin{array}{l}\text { - Persistent pulmonary symptoms are common after covid- } 19195 \\
\text { - In a } 3 \text { month follow-up study in China of covid-19 patients }(n=55), 71 \% \text { had radiologic abnormalities including interstitial thickening and fibrosis, } \\
\text { and } 25 \% \text { had impaired diffusing capacity for carbon monoxide at three months following discharge } 201 \\
\text { - An observational study from China of } 51 \text { covid-19 patients showed that } 45 \% \text { had abnormal computed tomography scans four weeks after } \\
\text { discharge }\end{array}$ \\
\hline Cardiac impairment & $\begin{array}{l}\text { - Evidence for long term sequelae from covid-19 has been noted, including evidence of myocardial inflammation on magnetic resonance imaging } \\
12-92 \text { days following infection }{ }^{203} 204\end{array}$ \\
\hline Neurologic impairment & $\begin{array}{l}\text { - While the occurrence of stroke due to covid-19 is relatively rare, other conditions including impairment of consciousness, encephalitis, seizure, } \\
\text { encephalopathy, and "brain fog" have been reported } 2-3 \text { months after initial illness onset } \\
\text { - Cognitive impairment is typically seen in } 30-80 \% \text { of patients who were in intensive care and includes memory loss as well as difficulty with } \\
\text { concentration, comprehension, and critical thinking }\end{array}$ \\
\hline
\end{tabular}

median adjusted odds ratio for hospital survival (OR 1.64, 1.14-2.35) and assumed probability of treatment effect to be neutral, which some experts feel is too high given prior negative trials. ${ }^{180}$ RECOVERY was a randomized adaptive platform open label trial $(n=2022$ tocilizumab, $n=2094$ usual care). Given the adaptive design, those who showed evidence for progressive disease $\left(\mathrm{saO}_{2}<92 \%\right.$ on room air and $\mathrm{C}$ reactive protein $>=75 \mathrm{mg} / \mathrm{L}$ ) up to 21 days after randomization were considered for tocilizumab. Twenty eight day mortality was 29\% (596/2022) for tocilizumab, and 33\% (694/2094) for usual care (rate ratio 0.86, confidence interval 0.77 to 0.96 ; $\mathrm{p}=0.007)$. The authors also reported a clear mortality benefit in those receiving corticosteroids in all prespecified subgroups $(27 \% v 33 \%$; rate ratio 0.80 ; confidence interval 0.70 to 0.90 ). The tocilizumab arm was less likely to reach composite endpoint of need for IMV or death $(33 \% v 38 \%$; risk ratio 0.85 , confidence interval 0.78 to $0.93 ; \mathrm{p}=0.0005) .{ }^{182}$ Given the other five trials ${ }^{175-179}$ did not show a significant mortality benefit or improvement in clinical outcomes, the results from RCTs for tocilizumab have been mixed. The largest trials ${ }^{180} 182$ report a modest mortality benefit and improvement in outcomes; however, adaptive trials are at risk of bias that can influence non-mortality outcomes. The reason for mixed results is unclear, and possible reasons include: earlier trials had inadequate power to detect a modest benefit, the necessity for corticosteroid use, or early use in critical illness is needed for tocilizumab to be effective.

\section{Convalescent plasma}

Convalescent plasma or plasma obtained from patients who have recovered from an infection have been used historically to treat infections. Treatment is hypothesized to work best when given early in the disease process before a patient develops an antibody response, and when it contains adequate concentrations of neutralizing antibodies. ${ }^{183}$ One large observational study analyzed data on convalescent plasma use among hospitalized patients at 2807 acute care facilities under the US FDA Expanded Access Program. ${ }^{184}$ Of the patients included, $52.3 \%$ were in intensive care and $27.5 \%$ were on mechanical ventilation. The 7 day mortality rate was $8.7 \%$ (95\% confidenceinterval $8.3 \%$ to $9.2 \%)$ in patients transfused within three days of covid-19 diagnosis but $11.9 \%$ (11.4\% to $12.2 \%)$ in those four or more days after diagnosis $(\mathrm{P}<0.001)$. The 30 day mortality was also lower in the patients transfused early $(21.6 \%$ versus $26.7 \%, \mathrm{P}<0.0001)$. The study reported that patients who received high IgG plasma had a lower 7 day mortality than those who received medium IgG plasma and low IgG plasma. However, the study used a semi-quantitative antibody assay, did not measure neutralizing antibody titers, and only compared early with late administration of convalescent plasma and convalescent plasma with different semi-quantitative levels of antibodies but not placebo. Eight RCTs have since evaluated convalescent plasma for the treatment of covid-19. Five of the studies had less than 100 patients in both arms and two had more than 200 patients in the convalescent plasma arm and 100 patients in the control arm. ${ }^{185}$ Most of the RCTs did not show a beneficial effect for mortality or clinical status, which had been seen in the observational studies. One RCT evaluated convalescent plasma with high anti-SARS-CoV2 IgG titers in older patients within 72 hours of mild covid-19 symptoms. In the convalescent plasma arm, $16.2 \%(13 / 80)$ progressed to severe respiratory diseases (respiratory rate $\geq 30$ or $\mathrm{O}_{2}$ sat $\left.<93 \%\right)^{186}$ compared with $31.2 \%(25 / 80)$, in a preplanned interim analysis. Early administration of high titer convalescent plasma may play a role in mild to moderate disease, but we need more data to 


\begin{tabular}{ll}
\hline Table $4 \mid$ Assessment of patients in post-ICU recovery clinics adapted to post-acute covid-19 patients \\
Instrument & Assessment \\
\hline Activities of Daily Living and Instrumental Activities of Daily Living & Functional status \\
\hline Physical therapy and occupational therapy evaluation & Functional assessment, mobility, strength \\
\hline European Quality of Life Five Dimension (EQ-5D) & Health related quality of life, mobility, pain \\
\hline Hospital Anxiety and Depression Scale (HADS) & Anxiety, depression \\
\hline Impact of Event Scale-Revised (IES-R) & Post-traumatic stress disorder \\
\hline Montreal Cognitive Assessment (MoCA) & Cognition \\
\hline Pulmonary function testing & Lung function \\
\hline 6 minute walk test & Lung function, functional status \\
\hline Chest radiograph & Lung parenchyma \\
\hline Echocardiogram and electrocardiogram & Cardiac function \\
\hline
\end{tabular}

delineate the exact role of convalescent plasma in the treatment of covid-19.

\section{Anticoagulation}

Patients with severe covid-19 are at increased risk for thrombosis ${ }^{58} 59$; however, no high quality evidence supports intermediate or full dose anticoagulation strategy over standard prophylactic anticoagulation. Clinical vigilance is needed in screening for thrombotic complications. D-dimers are associated with disease severity ${ }^{187}$ but at present no validated algorithms exist to guide anticoagulation regimens based on D-dimers. With the results of multiple RCTs ongoing, three linked trials investigating increased levels of anticoagulation paused enrollment for critically ill patients out of concern for futility and safety, ${ }^{188}$ but a recent press release suggested benefit to increased anticoagulation in the non-critically ill cohorts. ${ }^{189}$ The results of these and other ongoing studies should provide guidance on whether targeting a higher anticoagulation strategy in certain populations improves outcomes.

\section{Post-acute covid-19 complications}

Current estimates are that 91.5 million patients worldwide have recovered from SARS-CoV-2 infection. ${ }^{2}$ For those who survive covid-19, emerging reports have identified persistent symptoms beyond the acute phase of illness. These symptoms, which can affect multiple organ systems (table 3), are not due to persistent viral infection but instead sequelae of severe inflammation from the disease. ${ }^{209-211}$ "Post-acute covid-19" is defined as the presence of symptoms extending beyond three weeks, and "chronic covid-19" extends beyond 12 weeks. ${ }^{209}$ We know from studies before the pandemic that a high percentage of patients who require intensive care develop post-intensive care syndrome (PICS), which is the constellation of new or worsening physical and mental health and cognitive impairments that develop following critical illness. 190196212 These impairments often last beyond a year and have a profound impact on quality of life. ${ }^{213}$ Covid-19 patients who were in intensive care are particularly at risk ${ }^{196}$ to develop PICS given the high incidence of ARDS, prolonged mechanical ventilation, higher exposure to sedatives, higher incidence of delirium, limited physical therapy owing to concern for disease transmission, and constraints on social and emotional support owing to limited visits. ${ }^{214215}$

\section{Mitigation of post-ICU syndrome}

Prevention and mitigation of PICS can be accomplished by following the "ABCDEF" bundle ${ }^{216217}$ and other guidelines, ${ }^{218}$ which focus on managing pain, early ventilator liberation, assessing and treating delirium, appropriate usage of sedative agents, early mobility and exercise, and family engagement to prevent long term impairments. Early physical therapy and mobilization interventions ${ }^{208219}$ are paramount, and should be continued as an outpatient with home based physical therapy. 220221 Other interventions include ICU diaries, ${ }^{222} 223$ early psychological intervention, ${ }^{224}$ animal visitation, ${ }^{225}$ peer support groups for patients and families, 226227 and utilizing digital technology to bridge social distance. Healthcare providers should acknowledge should acknowledge the difficulty of covid-19, the unique stressors covid-19 patients and families are facing, and tailor their communication and behavior accordingly. ${ }^{215}$

\section{Importance of post-ICU recovery programs}

Patients who spent time in intensive care, especially patients with ARDS, are at high risk for PICS development. Without appropriate recognition, impairments go undiagnosed and can persist for months to years and profoundly affect quality of life. An interdisciplinary approach is essential to assist with diagnosis and management of critical illness recovery. Post-ICU recovery programs staffed by a team of providers (ie, pulmonologists, intensivists, pharmacists, advanced practice providers, nurses, physical and occupational therapists, respiratory therapists, social workers, case managers, and mental health providers) can diagnose and treat PICS impairments. ${ }^{228-230}$ These clinics also facilitate access to necessary subspecialties (tables 3, 4). The comprehensive approach of post-ICU clinics mirror the magnitude that critical illness affects multiple domains of a patient's health. By bringing together various subspecialty healthcare workers, these clinics promote mind, body, social, and spiritual recovery to survivors of critical illness. The need for ongoing ambulatory care for these vulnerable patients, also known as "long-haulers," is imperative. ${ }^{231}$ Long term longitudinal observational studies and clinical trials will be critical (box 1) to clarify the durability and extent of health consequences attributable to covid-19 and define best practices for covid-19 survivors. 


\section{Box 1: Covid-19 research questions}

- What are the pathophysiologic mechanisms for increased covid-19 severity in certain populations (ie, older adult populations, comorbidities, etc)?

- How does covid-19 related ARDS differ from classic ARDS?

- What is the best strategy for prevention of thrombotic complications in patients with covid-19 pneumonia?

- How does high flow nasal oxygen therapy compare with non-invasive ventilation as first line therapy for the treatment of respiratory failure in covid-19 pneumonia?

-What are the long term impacts of severe illness due to covid-19 related ARDS?

-What are the causes for persistent physical and cognitive impairments resulting from covid-19?
This article is made freely available for use in accordance with BM|'s website terms and conditions for the duration of the covid-19 pandemic or until otherwise determined by BMJ. You may use, download and print the article for any lawful, non-commercial purpose (including text and data mining) provided that all copyright notices and trade marks are retained.

1 National Institutes of Health. NIH covid-19 treatment guidelines. Clinical spectrum of SARS-CoV-2 Infection. 2020. https://www. covid19treatmentguidelines.nih.gov/overview/clinical-spectrum

2 Dong E, Du H, Gardner L. An interactive web-based dashboard to track COVID-19 in real time. Lancet Infect Dis 2020;20:533-4. doi:10.1016/S1473-3099(20)30120-1

3 Grasselli G, Zangrillo A, Zanella A, et al, COVID-19 Lombardy ICU Network. Baseline characteristics and outcomes of 1591 patients infected with SARS-CoV-2 admitted to ICUs of the Lombardy region, Italy. JAMA 2020;323:1574-81. doi:10.1001/jama.2020.5394

4 Richardson S, Hirsch JS, Narasimhan M, et al, the Northwell COVID-19 Research Consortium. Presenting characteristics, comorbidities, and outcomes among 5700 patients hospitalized with covid-19 in the New York City Area. JAMA 2020;323:2052-9. doi:10.1001/ jama.2020.6775

In formulating this review, we considered guidelines that provide recommendations on the management of covid-19 from the Infectious Diseases Society of America, ${ }^{232} 233$ World Health Organization, ${ }^{234}$ Society of Critical Care Medicine, ${ }^{235}$ and the National Institutes of Health. ${ }^{236}$ We selected these guidelines because of their recommendations for patients with covid-19 pneumonia, which included management and molecular diagnostics. We prioritized guidelines that used explicit methodology, which stated how searches were done systematically, how synthesis (meta-analysis) was performed, and how the evidence was appraised using a priori criteria. Additionally, guidelines for management of tracheostomy ${ }^{160} 161$ and respiratory failure ${ }^{120}$ are included in the respiratory care section.

\section{Conclusion}

Remarkable advances have been made in a short period in the treatment of covid-19 pneumonia, including the development of drug treatments that improve mortality and recovery from illness. As more clinical and mechanistic data emerge on CARDS, tailored therapy can be designed to further improve outcomes. Management of respiratory failure is guided by principles of management for classic ARDS. Despite these promising developments, including the development of vaccines, covid-19 will continue to have an impact on healthcare systems as thousands of patients recover from critical illness. An integrated therapeutic approach to mitigate the adverse physical and mental health effects of covid-19 pneumonia is essential.

Acknowledgments: The authors would like to thank Eduardo MirelesCabodevila, Abhijit Duggal, and Robert L Chatburn for their insightful review of the manuscript.

Contributors: All authors defined intellectual content, conducted literature research, acquired data, and participated in manuscript preparation, editing, and critical review. AHA is the named guarantor

Competing interests: We have read and understood the BMJ policy on declaration of interests and declare the following interests: UH reports receiving royalties from Wolters Kluwer Health for his work as section editor for UpToDate. No other disclosures were reported.

Patient involvement: No patients were directly involved in the creation of this article.

Grant Support: This work was supported by NIH grants (K08 HL133380 and R01 HL 155064) to RGSProvenance and peer review: Commissioned; externally peer reviewed.

Docherty AB, Harrison EM, Green CA, et al, ISARIC4C investigators. Features of 20133 UK patients in hospital with covid-19 using the ISARIC WHO Clinical Characterisation Protocol: prospective observational cohort study. BMJ 2020;369:m1985. doi:10.1136/ bmj.m1985

6 Garg S, Kim L, Whitaker M, et al. Hospitalization rates and characteristics of patients hospitalized with laboratory-confirmed coronavirus disease 2019-covid-NET, 14 States, March 1-30, 2020. MMWR Morb Mortal Wkly Rep 2020;69:458-64. doi:10.15585/ mmwr.mm6915e3

7 Torres Acosta MA, Singer BD. Pathogenesis of COVID-19induced ARDS: implications for an ageing population. Eur Respir / 2020:56:2002049. doi:10.1183/13993003.02049-2020

8 Yang Y, Zhao Y, Zhang F, Zhang L, Li L. covid-19 in elderly adults: clinical features, molecular mechanisms, and proposed strategies. Aging Dis 2020:11:1481-95. doi:10.14336/AD.2020.0903

9 Ellinghaus D, Degenhardt F, Bujanda L, et al, Severe Covid-19 GWAS Group. Genomewide association study of severe covid-19 with Respiratory Failure. N Engl J Med 2020;383:1522-34. doi:10.1056/ NEJMoa2020283

10 Yang $X, Y u Y, X u$ J, et al. Clinical course and outcomes of critically ill patients with SARS-CoV-2 pneumonia in Wuhan, China: a single-centered, retrospective, observational study. Lancet Respir Med 2020;8:475-81. doi:10.1016/S2213-2600(20)30079-5

11 Lauer SA, Grantz KH, Bi Q, et al. The incubation period of coronavirus disease 2019 (covid-19) from publicly reported confirmed cases: estimation and application. Ann Intern Med 2020;172:577-82. doi:10.7326/M20-0504

12 Hu B, Guo H, Zhou P, Shi Z-L. Characteristics of SARS-CoV-2 and COVID-19. Nat Rev Microbiol 2020;6:1-14.

13 Tzotzos SJ, Fischer B, Fischer H, Zeitlinger M. Incidence of ARDS and outcomes in hospitalized patients with COVID-19: a global literature survey. Crit Care 2020;24:516. doi:10.1186/s13054-020-03240-7

14 Petrilli CM, Jones SA, Yang J, et al. Factors associated with hospital admission and critical illness among 5279 people with coronavirus disease 2019 in New York City: prospective cohort study. BMJ 2020;369:m1966. doi:10.1136/bmj.m1966

15 Horwitz LI, Jones SA, Cerfolio RJ, et al. Trends in covid-19 riskadjusted mortality rates. J Hosp Med 2021;16:90-2. doi:10.12788/ jhm.3552

16 Dennis JM, McGovern AP, Vollmer SJ, Mateen BA. Improving survival of critical care patients with coronavirus disease 2019 in England a national cohort study, March to June 2020. Crit Care Med 2021;49:209-14. doi:10.1097/CCM.0000000000004747

17 Lan J, Ge J, Yu J, et al. Structure of the SARS-CoV-2 spike receptorbinding domain bound to the ACE2 receptor. Nature 2020;581:215 20. doi:10.1038/s41586-020-2180-5

18 Meyerowitz EA, Richterman A, Gandhi RT, Sax PE. Transmission of SARS-CoV-2: a review of viral, host, and environmental factors. Ann Intern Med 2020, doi:10.7326/M20-5008

19 da Costa VG, Moreli ML, Saivish MV. The emergence of SARS, MERS and novel SARS-2 coronaviruses in the 21st century. Arch Virol 2020;165:1517-26. doi:10.1007/s00705-020-04628-0

20 Shang J, Ye G, Shi K, et al. Structural basis of receptor recognition by SARS-CoV-2. Nature 2020;581:221-4. doi:10.1038/s41586-0202179-y

21 Zhang L, Lin D, Sun X, et al. Crystal structure of SARS-CoV-2 main protease provides a basis for design of improved a-ketoamide inhibitors. Science 2020;368:409-12. doi:10.1126/science. abb3405

22 Walls AC, Park Y-J, Tortorici MA, Wall A, McGuire AT, Veesler D. Structure, function, and antigenicity of the SARS-CoV-2 spike 
glycoprotein. Cell 2020;181:281-292.e6. doi:10.1016/j. cell.2020.02.058

23 Siu YL, Teoh KT, Lo J, et al. The M, E, and N structural proteins of the severe acute respiratory syndrome coronavirus are required for efficient assembly, trafficking, and release of virus-like particles. J Virol 2008:82:11318-30. doi:10.1128/JVI.01052-08

24 Satarker S, Nampoothiri M. Structural proteins in severe acute respiratory syndrome coronavirus-2. Arch Med Res 2020;51:482-91. doi:10.1016/i.arcmed.2020.05.012

25 Huang Y, Yang C, Xu XF, Xu W, Liu SW. Structural and functional properties of SARS-CoV-2 spike protein: potential antivirus drug development for COVID-19. Acta Pharmacol Sin 2020;41:1141-9. doi:10.1038/s41401-020-0485-4

26 Duan L, Zheng Q, Zhang H, Niu Y, Lou Y, Wang H. The SARSCoV-2 spike glycoprotein biosynthesis, structure, function, and antigenicity: implications for the design of spike-based vaccine immunogens. Front Immunol 2020;11:576622. doi:10.3389/ fimmu.2020.576622

27 Hu Y, Wen J, Tang L, et al. The M protein of SARS-CoV: basic structural and immunological properties. Genomics Proteomics Bioinformatics 2003;1:118-30. doi:10.1016/S16720229(03)01016-7

28 Singh Tomar PP, Arkin IT. SARS-CoV-2 E protein is a potential ion channel that can be inhibited by Gliclazide and Memantine. Biochem Biophys Res Commun 2020;530:10-4. doi:10.1016/j. bbrc.2020.05.206

29 Sarkar M, Saha S. Structural insight into the role of novel SARS-CoV-2 E protein: A potential target for vaccine development and other therapeutic strategies. PLoS One 2020;15:e0237300. doi:10.1371/ journal.pone.0237300

30 Dutta NK, Mazumdar K, Gordy JT. The nucleocapsid protein of SARSCoV-2: a target for vaccine development. J Virol 2020;94:e0064720. doi:10.1128/JVI.00647-20

31 Lopez-Rincon A, Perez-Romero CA, Tonda A, et al. Design of specific primer sets for the detection of B.1.1.7, B.1.351 and P.1 SARS-CoV-2 variants using deep learning.bioRxiv [Preprint] 2021. doi:10.1101/2021.01.20.427043

32 Vrancken B, Dellicour S, Smith DM, Chaillon A. Phylogenetic analyses of SARS-CoV-2 B.1.1.7 lineage suggest a single origin followed by multiple exportation events versus convergent evolution.bioRxiv [Preprint] 2021. doi:10.1101/2021.01.12.426373

33 Centers for Disease Control and Prevention. Emerging SARS-CoV-2 variants. 2021. https://www.cdc.gov/coronavirus/2019-ncov/ more/science-and-research/scientific-brief-emerging-variants. html

34 Starr TN, Greaney AJ, Addetia A, et al. Prospective mapping of viral mutations that escape antibodies used to treat COVID-19. Science 2021:eabf9302. doi:10.1126/science.abf9302

35 Wang Q, Zhang Y, Wu L, et al. Structural and functional basis of SARS CoV-2 entry by using human ACE2. Cell 2020;181:894-904.e9. doi:10.1016/j.cell.2020.03.045

36 Samavati L, Uhal BD. ACE2, much more than just a receptor for SARS-COV-2. Front Cell Infect Microbiol 2020;10:317. doi:10.3389/ fcimb.2020.00317

37 Yuki K, Fujiogi M, Koutsogiannaki S. COVID-19 pathophysiology: A review. Clin Immunol 2020;215:108427. doi:10.1016/j. clim.2020.108427

38 Swärd P, Edsfeldt A, Reepalu A, Jehpsson L, Rosengren BE, Karlsson MK. Age and sex differences in soluble ACE2 may give insights for COVID-19. Crit Care 2020;24:221. doi:10.1186/s13054-02002942-2

39 Hoffmann M, Kleine-Weber H, Schroeder S, et al. SARS-CoV-2 cell entry depends on ACE2 and TMPRSS2 and is blocked by clinically proven protease inhibitor. Cell 2020;181:271-280.e8. doi:10.1016/j.cell.2020.02.052

40 Ma D, Chen C-B, Jhanji V et al. Expression of SARS-CoV-2 receptor ACE2 and TMPRSS2 in human primary conjunctival and pterygium cell lines and in mouse cornea. Eye (Lond) 2020;34:1212-9. doi:10.1038/s41433-020-0939-4

41 Bao R, Hernandez K, Huang L, Luke JJ. ACE2 and TMPRSS2 expression by clinical, HLA, immune, and microbial correlates across 34 human cancers and matched normal tissues: implications for SARS-CoV-2 COVID-19. I Immunother Cancer 2020;8:e001020. doi:10.1136/jitc2020-001020

42 Bergmann CC, Silverman RH. COVID-19: Coronavirus replication, pathogenesis, and therapeutic strategies. Cleve Clin I Med 2020;87:321-7. doi:10.3949/ccjm.87a.20047

43 Blanco-Melo D, Nilsson-Payant BE, Liu W-C, et al. Imbalanced host response to SARS-CoV-2 drives development of covid-19. Cell 2020;181:1036-1045.e9. doi:10.1016/j.cell.2020.04.026

44 Guo L, Ren L, Yang S, et al. Profiling early humoral response to diagnose novel coronavirus disease (covid-19). Clin Infect Dis 2020:71:778-85. doi:10.1093/cid/ciaa310

45 Woodruff MC, Ramonell RP, Nguyen DC, et al. Extrafollicular B cell responses correlate with neutralizing antibodies and morbidity in
COVID-19. Nat Immunol 2020;21:1506-16. doi:10.1038/s41590020-00814-z

46 Long Q-X, Tang X-J, Shi Q-L, et al. Clinical and immunological assessment of asymptomatic SARS-CoV-2 infections. Nat Med 2020;26:1200-4. doi:10.1038/s41591-020-0965-6

47 Ren L, Fan G, Wu W, et al. Antibody responses and clinical outcomes in adults hospitalized with severe coronavirus disease 2019 (covid-19): a post hoc analysis of LOTUS China trial. Clin Infect Dis 2020. doi:10.1093/cid/ciaa1247

48 Tan L, Wang Q, Zhang D, et al. Lymphopenia predicts disease severity of COVID-19: a descriptive and predictive study. Signal Transduct Target Ther 2020;5:33. doi:10.1038/s41392-020-0148-4

49 Rydyznski Moderbacher C, Ramirez SI, Dan JM, et al. Antigen-specific adaptive immunity to SARS-CoV- 2 in acute covid-19 and associations with age and disease severity. Cell 2020;183:996-1012.e19. doi:10.1016/i.cell.2020.09.038

50 Goronzy JJ, Weyand CM. Mechanisms underlying T cell ageing. Nat Rev Immunol 2019;19:573-83. doi:10.1038/s41577-019-0180-1

51 Wilk AJ, Rustagi A, Zhao NQ, et al. A single-cell atlas of the peripheral immune response in patients with severe COVID-19. Nat Med 2020;26:1070-6. doi:10.1038/s41591-020-0944-y

52 Del Valle DM, Kim-Schulze S, Huang H-H, et al. An inflammatory cytokine signature predicts COVID-19 severity and survival. Nat Med 2020;26:1636-43. doi:10.1038/s41591-020-1051-9

53 Leisman DE, Ronner L, Pinotti R, et al. Cytokine elevation in severe and critical COVID-19: a rapid systematic review, meta-analysis, and comparison with other inflammatory syndromes. Lancet Respir Med 2020;8:1233-44. doi:10.1016/S2213-2600(20)30404-5

54 RECOVERY Collaborative Group. Dexamethasone in hospitalized patients with Covid-19-preliminary report. N Engl / Med 2020; doi:10.1056/NEJMoa2021436.

55 Wilson JG, Simpson LJ, Ferreira A-M, et al. Cytokine profile in plasma of severe COVID-19 does not differ from ARDS and sepsis. $/ C l$ Insight 2020;5:140289. doi:10.1172/jci.insight.140289

56 Maiese A, Manetti AC, La Russa R, et al. Autopsy findings in COVID19-related deaths: a literature review. Forensic Sci Med Pathol 2020. doi:10.1007/s12024-020-00310-8

57 Arrossi AV, Farver C. The pulmonary pathology of COVID-19. Cleve Clin J Med 2020. doi:10.3949/ccim.87a.ccc063

58 Malas MB, Naazie IN, Elsayed N, Mathlouthi A, Marmor R, Clary B. Thromboembolism risk of COVID-19 is high and associated with a higher risk of mortality: A systematic review and metaanalysis. EClinicalMedicine 2020;29:100639. doi:10.1016/j. eclinm.2020.100639

59 Chi G, Lee JJ, Jamil A, et al. Venous thromboembolism among hospitalized patients with covid-19 undergoing thromboprophylaxis: a systematic review and meta-analysis. J Clin Med 2020;9:2489. doi:10.3390/jcm9082489

60 Ranieri VM, Rubenfeld GD, Thompson BT, et al, ARDS Definition Task Force. Acute respiratory distress syndrome: the Berlin Definition. JAMA 2012;307:2526-33.

61 Habashi NM, Camporota L, Gatto LA, Nieman GF. Functional pathophysiology of SARS-CoV-2 induced acute lung injury and clinical implications. J Appl Physiol (1985) 2021. doi:10.1152/ japplphysiol.00742.2020

62 Chu DK, Kim LHY, Young PJ, et al. Mortality and morbidity in acutely il adults treated with liberal versus conservative oxygen therapy (IOTA) a systematic review and meta-analysis. Lancet 2018;391:1693-705. doi:10.1016/S0140-6736(18)30479-3

63 Siemieniuk RAC, Chu DK, Kim LH, et al. Oxygen therapy for acutely ill medical patients: a clinical practice guideline. BMJ 2018;363:k4169. doi:10.1136/bmj.k4169

64 Barrot L, Asfar P, Mauny F, et al, LOCO2 Investigators and REVA Research Network. Liberal or conservative oxygen therapy for acute respiratory distress syndrome. N Engl J Med 2020;382:999-1008. doi:10.1056/NEIMoa1916431

65 Brower RG, Matthay MA, Morris A, Schoenfeld D, Thompson BT, Wheeler A, Acute Respiratory Distress Syndrome Network. Ventilation with lower tidal volumes as compared with traditional tidal volumes for acute lung injury and the acute respiratory distress syndrome. N Engl J Med 2000;342:1301-8. doi:10.1056/ NEJM200005043421801

66 Slutsky AS, Ranieri VM. Ventilator-induced lung injury. N Engl/ Med 2013;369:2126-36. doi:10.1056/NEJMra1208707

67 Mascheroni D, Kolobow T, Fumagalli R, Moretti MP, Chen V, Buckhold $D$. Acute respiratory failure following pharmacologically induced hyperventilation: an experimental animal study. Intensive Care Med 1988;15:8-14. doi:10.1007/BF00255628

68 Carteaux G, Millán-Guilarte T, De Prost N, et al. Failure of noninvasive ventilation for de novo acute hypoxemic respiratory failure: role of tidal volume. Crit Care Med 2016;44:282-90. doi:10.1097/ CCM.0000000000001379

69 Brochard L, Slutsky A, Pesenti A. Mechanical ventilation to minimize progression of lung injury in acute respiratory failure. Am J Respir Crit Care Med 2017;195:438-42. doi:10.1164/rccm.201605-1081CP 
70 Amato MBP, Meade MO, Slutsky AS, et al. Driving pressure and survival in the acute respiratory distress syndrome. $N$ Engl Med 2015:372:747-55 doi:10.1056/NEIMsa1410639

71 Chatburn RL, van der Staay M. Driving pressure or tidal pressure: what a difference a name makes. Respir Care 2019;64:1176-9. doi:10.4187/respcare.07233

72 Brower RG, Lanken PN, Maclntyre N, et al, National Heart, Lung, and Blood Institute ARDS Clinical Trials Network. Higher versus lower positive end-expiratory pressures in patients with the acute respiratory distress syndrome. N Engl J Med 2004;351:327-36. doi:10.1056/NEJMoa032193

73 Meade MO, Cook DJ, Guyatt GH, et al, Lung Open Ventilation Study Investigators. Ventilation strategy using low tidal volumes, recruitment maneuvers, and high positive end-expiratory pressure for acute lung injury and acute respiratory distress syndrome: a randomized controlled trial. JAMA 2008;299:637-45. doi:10.1001/ jama.299.6.637

74 Cavalcanti AB, Suzumura ÉA, Laranjeira LN, et al, Writing Group for the Alveolar Recruitment for Acute Respiratory Distress Syndrome Trial (ART) Investigators. Effect of lung recruitment and titrated positive end-expiratory pressure (PEEP) vs low PEEP on mortality in patients with acute respiratory distress syndrome: randomized clinical trial. JAMA 2017;318:1335-45. doi:10.1001/ jama.2017.14171

75 Chen L, Del Sorbo L, Grieco DL, et al. Potential for lung recruitment estimated by the recruitment-to-inflation ratio in acute respiratory distress syndrome. A clinical trial. Am J Respir Crit Care Med 2020;201:178-87. doi:10.1164/rccm.201902-03340C

76 Pan C, Chen L, Lu C, et al. Lung recruitability in covid-19-associated acute respiratory distress syndrome: a single-center observational study. Am J Respir Crit Care Med 2020;201:1294-7. doi:10.1164/ rccm.202003-0527LE

77 Grasso S, Mirabella L, Murgolo F, et al. Effects of positive end-expiratory pressure in "high compliance" severe acute respiratory syndrome coronavirus 2 acute respiratory distress syndrome. Crit Care Med 2020;48:e1332-6. doi:10.1097/ CCM.0000000000004640

78 Karbing DS, Panigada M, Bottino N, et al. Changes in shunt, ventilation/perfusion mismatch, and lung aeration with PEEP in patients with ARDS: a prospective single-arm interventional study. Crit Care 2020;24:111. doi:10.1186/s13054-020-2834-6

79 Guérin C, Reignier J, Richard J-C, et al, PROSEVA Study Group. Prone positioning in severe acute respiratory distress syndrome. N Engl/ Med 2013;368:2159-68. doi:10.1056/NEJMoa1214103

80 Ho ATN, Patolia S, Guervilly C. Neuromuscular blockade in acute respiratory distress syndrome: a systematic review and metaanalysis of randomized controlled trials. J Intensive Care 2020;8:12. doi:10.1186/s40560-020-0431-z

81 Gattinoni L, Coppola S, Cressoni M.Covid-19 does not lead to a "typical" acute respiratory distress syndrome. Am / Respir Crit Care Med 2020;201:1299-300.

82 Fan E, Beitler JR, Brochard L, et al. COVID-19-associated acute respiratory distress syndrome: is a different approach to management warranted?Lancet Respir Med 2020;8:816-21. doi:10.1016/S2213-2600(20)30304-0

83 Tobin MJ, Laghi F, Jubran A. P-SILI is not justification for intubation of COVID-19 patients. Ann Intensive Care 2020;10:105. doi:10.1186/ s13613-020-00724-1

84 Schmidt M, Hajage D, Lebreton G, et al, Groupe de Recherche Clinique en REanimation et Soins intensifs du Patient en Insuffisance Respiratoire aiguE (GRC-RESPIRE) Sorbonne Université, ParisSorbonne ECMO-COVID investigators. Extracorporeal membrane oxygenation for severe acute respiratory distress syndrome associated with COVID-19: a retrospective cohort study. Lancet Respir Med 2020;8:1121-31. doi:10.1016/S2213-2600(20)30328-3

85 Ferrando C, Suarez-Sipmann F, Mellado-Artigas R, et al, COVID-19 Spanish ICU Network. Clinical features, ventilatory management, and outcome of ARDS caused by COVID-19 are similar to other causes of ARDS. Intensive Care Med 2020;46:2200-11. doi:10.1007/s00134020-06192-2

86 Spoletini G, Alotaibi M, Blasi F, Hill NS. Heated humidified highflow nasal oxygen in adults: mechanisms of action and clinical implications. Chest 2015;148:253-61. doi:10.1378/chest.14-2871

87 Möller W, Feng S, Domanski U, et al. Nasal high flow reduces dead space. J Appl Physiol (1985) 2017;122:191-7. doi:10.1152/ japplphysiol.00584.2016

88 Parke RL, McGuinness SP. Pressures delivered by nasal high flow oxygen during all phases of the respiratory cycle. Respir Care 2013:58:1621-4. doi:10.4187/respcare.02358

89 Rochwerg B, Granton D, Wang DX, et al. High flow nasal cannula compared with conventional oxygen therapy for acute hypoxemic respiratory failure: a systematic review and meta-analysis. Intensive Care Med 2019:45:563-72. doi:10.1007/s00134-019-05658-2

90 Agarwal A, Basmaji J, Muttalib F, et al. High-flow nasal cannula for acute hypoxemic respiratory failure in patients with
COVID-19: systematic reviews of effectiveness and its risks of aerosolization, dispersion, and infection transmission. Can J Anaesth 2020;67:1217-48. doi:10.1007/s12630-020-01740-2

91 Roca O, Caralt B, Messika J, et al. An index combining respiratory rate and oxygenation to predict outcome of nasal high-flow therapy. Am J Respir Crit Care Med 2019;199:1368-76. doi:10.1164/ rccm.201803-05890C

92 Kang BJ, Koh Y, Lim C-M, et al. Failure of high-flow nasal cannula therapy may delay intubation and increase mortality. Intensive Care Med 2015;41:623-32. doi:10.1007/s00134-015-3693-5

93 Calligaro GL, Lalla U, Audley G, et al. The utility of high-flow nasal oxygen for severe COVID-19 pneumonia in a resourceconstrained setting: A multi-centre prospective observational study. EClinicalMedicine 2020;28:100570. doi:10.1016/j. eclinm.2020.100570

94 Ferrando C, Mellado-Artigas R, Gea A, et al, COVID-19 Spanish ICU Network. Awake prone positioning does not reduce the risk of intubation in COVID-19 treated with high-flow nasal oxygen therapy: a multicenter, adjusted cohort study. Crit Care 2020;24:597. doi:10.1186/s13054-020-03314-6

95 Zucman N, Mullaert J, Roux D, Roca O, Ricard J-D, Contributors. Prediction of outcome of nasal high flow use during COVID19-related acute hypoxemic respiratory failure. Intensive Care Med 2020;46:1924-6. doi:10.1007/s00134-020-06177-1

96 Xia J, Zhang Y, Ni L, et al. High-flow nasal oxygen in coronavirus disease 2019 patients with acute hypoxemic respiratory failure: a multicenter, retrospective cohort study. Crit Care Med 2020;48:e1079-86. doi:10.1097/CCM.0000000000004558

97 Panadero C, Abad-Fernández A, Rio-Ramirez MT, et al. High-flow nasal cannula for Acute Respiratory Distress Syndrome (ARDS) due to COVID-19. Multidiscip Respir Med 2020;15:693. doi:10.4081/ mrm.2020.693

98 Vianello A, Arcaro G, Molena B, et al. High-flow nasal cannula oxygen therapy to treat patients with hypoxemic acute respiratory failure consequent to SARS-CoV-2 infection. Thorax 2020;75:998-1000. doi:10.1136/thoraxinl-2020-214993

99 Guy T, Créac'hcadec A, Ricordel C, et al. High-flow nasal oxygen: a safe, efficient treatment for COVID-19 patients not in an ICU. Eur Respir / 2020;56:2001154. doi:10.1183/13993003.01154-2020

100 Duan J, Chen B, Liu X, et al. Use of high-flow nasal cannula and noninvasive ventilation in patients with COVID-19: A multicenter observational study. Am J Emerg Med 2020;S07356757(20)30666-5. doi:10.1016/i.ajem.2020.07.071

101 Wang K, Zhao W, Li J, Shu W, Duan J. The experience of high-flow nasal cannula in hospitalized patients with 2019 novel coronavirusinfected pneumonia in two hospitals of Chongqing, China. Ann Intensive Care 2020;10:37. doi:10.1186/s13613-020-00653-z

102 Demoule A, Vieillard Baron A, Darmon M, et al. High-flownasal cannula in critically ill patients with severe covid-19. Am / Respir Crit Care Med 2020;202:1039-42.

103 Tran K, Cimon K, Severn M, Pessoa-Silva CL, Conly J. Aerosol generating procedures and risk of transmission of acute respiratory infections to healthcare workers: a systematic review. PLoS One 2012;7:e35797. doi:10.1371/journal.pone.0035797

104 Dhand R, Li J. Coughs and sneezes: their role in transmission of respiratory viral infections, including SARS-CoV-2. Am J Respir Crit Care Med 2020;202:651-9. doi:10.1164/rccm.202004-1263PP

105 Gaeckle NT, Lee J, Park Y, Kreykes G, Evans MD, Hogan CJJr. Aerosol generation from the respiratory tract with various modes of oxygen delivery. Am J Respir Crit Care Med 2020;202:1115-24. doi:10.1164/rccm.202006-23090C

106 Hui DS, Chow BK, Lo T, et al. Exhaled air dispersion during high-flow nasal cannula therapy versus CPAP via different masks. Eur Respir / 2019;53:1802339. doi:10.1183/13993003.02339-2018

107 Li J, Ehrmann S. High-flow aerosol-dispersing versus aerosolgenerating procedures. Am J Respir Crit Care Med 2020;202:106971. doi:10.1164/rccm.202008-3317ED

108 Gershengorn HB, Hu Y, Chen J-T, et al. The impact of high-flow nasal cannula use on patient mortality and the availability of mechanical ventilators in covid-19. Ann Am Thorac Soc 2020. doi:10.1513/ AnnalsATS.202007-8030C

109 Mellado-Artigas R, Ferreyro BL, Angriman F, et al. COVID-19 Spanish ICU Network. High-flow nasal oxygen in patients with COVID-19associated acute respiratory failure. Crit Care 2021;25:58.

110 Berbenetz N, Wang Y, Brown J, et al. Non-invasive positive pressure ventilation (CPAP or bilevel NPPV) for cardiogenic pulmonary oedema[-CD.]. Cochrane Database Syst Rev 2019;4:CD005351. doi:10.1002/14651858.CD005351.pub4

111 Osadnik CR, Tee VS, Carson-Chahhoud KV, Picot J, Wedzicha JA, Smith BJ. Non-invasive ventilation for the management of acute hypercapnic respiratory failure due to exacerbation of chronic obstructive pulmonary disease[-CD.]. Cochrane Database Syst Rev 2017;7:CD004104. doi:10.1002/14651858.CD004104.pub4

112 Frat JP, Ragot S, Coudroy R, et al, REVA network. Predictors of intubation in patients with acute hypoxemic respiratory failure 
treated with a noninvasive oxygenation strategy. Crit Care Med 2018;46:208-15. doi:10.1097/CCM.0000000000002818

113 Xu XP, Zhang XC, Hu SL, et al. Noninvasive ventilation in acute hypoxemic nonhypercapnic respiratory failure: a systematic review and meta-analysis. Crit Care Med 2017;45:e727-33. doi:10.1097/ CCM.0000000000002361

114 Kondo Y, Kumasawa J, Kawaguchi A, Seo R, Nango E, Hashimoto S. Effects of non-invasive ventilation in patients with acute respiratory failure excluding post-extubation respiratory failure, cardiogenic pulmonary edema and exacerbation of COPD: a systematic review and meta-analysis. J Anesth 2017;31:714-25. doi:10.1007/ s00540-017-2389-0

115 David-João PG, Guedes MH, Réa-Neto Á, Chaiben VBO, Baena CP. Noninvasive ventilation in acute hypoxemic respiratory failure: $A$ systematic review and meta-analysis. J Crit Care 2019;49:84-91. doi:10.1016/j.jcrc.2018.10.012

116 Bellani G, Laffey JG, Pham T, et al, LUNG SAFE Investigators, ESICM Trials Group. Noninvasive ventilation of patients with acute respiratory distress syndrome. Insights from the LUNG SAFE study. Am I Respir Crit Care Med 2017;195:67-77. doi:10.1164/rccm.201606-13060C

117 Frat J-P, Ragot S, Girault C, et al, REVA network. Effect of non-invasive oxygenation strategies in immunocompromised patients with severe acute respiratory failure: a post-hoc analysis of a randomised trial. Lancet Respir Med 2016;4:646-52. doi:10.1016/S22132600(16)30093-5

118 Ferreyro BL, Angriman F, Munshi L, et al. Association of noninvasive oxygenation strategies with all-cause mortality in adults with acute hypoxemic respiratory failure: a systematic review and meta-analysis. JAMA 2020;324:57-67. doi:10.1001/jama.2020.9524

119 Alhazzani W, Møller MH, Arabi YM, et al. Surviving sepsis campaign: guidelines on the management of critically ill adults with coronavirus disease 2019 (covid-19). Crit Care Med 2020;48:e440-69. doi:10.1097/CCM.0000000000004363

120 Pfeifer M, Ewig S, Voshaar T, et al, German Respiratory Society. Position paper for the state of the art application of respiratory support in patients with covid-19. Pneumologie 2020;74:337-57.

121 Hua J, Qian C, Luo Z, Li Q, Wang F. Invasive mechanical ventilation in COVID-19 patient management: the experience with 469 patients in Wuhan. Crit Care 2020;24:348. doi:10.1186/s13054-020-03044-9

122 Guan W-J, Ni Z-Y, Hu Y, et al, China Medical Treatment Expert Group for Covid-19. Clinical characteristics of coronavirus disease 2019 in China. N Engl J Med 2020;382:1708-20. doi:10.1056/ NEJMoa2002032

123 Wang T, Tang C, Chen R, et al. Clinical features of coronavirus disease 2019 patients with mechanical ventilation: a nationwide study in China. Crit Care Med 2020:48:e809-12.

124 Guan L, Zhou L, Le Grange JM, Zheng Z, Chen R. Non-invasive ventilation in the treatment of early hypoxemic respiratory failure caused by COVID-19: considering nasal CPAP as the first choice. Crit Care 2020;24:333. doi:10.1186/s13054-020-03054-7

125 Duca A, Memaj I, Zanardi F, et al. Severity of respiratory failure and outcome of patients needing a ventilatory support in the Emergency Department during Italian novel coronavirus SARS-CoV2 outbreak: Preliminary data on the role of Helmet CPAP and Non-Invasive Positive Pressure Ventilation. EClinicalMedicine 2020;24:100419. doi:10.1016/i.eclinm.2020.100419

126 Sivaloganathan AA, Nasim-Mohi M, Brown MM, et al, University Hospital Southampton Critical Care and Respiratory Medicine Teams and the REACT investigators, UHS Critical Care Clinical Team, UHS Respiratory Clinical Team, REACT Investigators. Noninvasive ventilation for COVID-19-associated acute hypoxaemic respiratory failure: experience from a single centre. $\mathrm{Br}$ J Anaesth 2020;125:e368-71. doi:10.1016/j.bja.2020.07.008

127 Oranger M, Gonzalez-Bermejo J, Dacosta-Noble P, et al. Continuous positive airway pressure to avoid intubation in SARS-CoV-2 pneumonia: a two-period retrospective case-control study. Eur Respir / 2020;56:2001692. doi:10.1183/13993003.01692-2020

128 Burns GP, Lane ND, Tedd HM, et al. Improved survival following ward-based non-invasive pressure support for severe hypoxia in a cohort of frail patients with COVID-19: retrospective analysis from a UK teaching hospital. BMJ Open Respir Res 2020;7:e000621. doi:10.1136/bmjresp-2020-000621

129 Zheng Y, Sun L-J, Xu M, et al. Clinical characteristics of 34 COVID-19 patients admitted to intensive care unit in Hangzhou, China. J Zhejiang Univ Sci B 2020;21:378-87. doi:10.1631/jzus.B2000174

130 Aliberti S, Radovanovic D, Billi F, et al. Helmet CPAP treatment in patients with COVID-19 pneumonia: a multicentre cohort study. Eur Respir / 2020;56:2001935. doi:10.1183/13993003.01935-2020

131 Bellani G, Graselli G, Cecconi M, et al. COVID-19 Lombardy ICU Network. Noninvasive Ventilatory Support of COVID-19 Patients Outside the Intensive Care Units (WARd-COVID). Ann Am Thorac Soc 2021; doi:10.1513/AnnalsATS.202008-10800C

132 Zhang Q, Shen J, Chen L, et al. Timing of invasive mechanic ventilation in critically ill patients with coronavirus disease 2019 . J Trauma Acute Care Surg 2020;89:1092-8.
133 Potalivo A, Montomoli J, Facondini F, et al. Sixty-day mortality among 520 Italian hospitalized COVID-19 patients according to the adopted ventilatory strategy in the context of an integrated multidisciplinary clinical organization: a population-based cohort study. Clin Epidemiol 2020;12:1421-31.

134 Diaz de Teran T, Gonzalez Martinez M, Banfi P, et al. Management of patients with severe acute respiratory failure due to SARS CoV-2 pneumonia with non-invasive ventilatory support outside intensive care unit. Minerva Med 2021; doi:10.23736/S00264806.21.07134-2.

135 Dupuis C, Bouadma L, de Montmollin E, et al. Association between early invasive mechanical ventilation and day-60 mortality in acute hypoxemic respiratory failure related to coronavirus disease-2019 pneumonia. Crit Care Explor 2021;3:e0329.

136 Daniel P, Mecklenburg M, Massiah C, et al. Non-invasive positive pressure ventilation versus endotracheal intubation in treatmen of COVID-19 patients requiring ventilatory support. Am J Emerg Med 2021;43:103-8.

137 Schenck EJ, Hoffman K, Goyal P, et al. Respiratory mechanics and gas exchange in covid-19-associated respiratory failure. Ann Am Thorac Soc 2020;17:1158-61. doi:10.1513/AnnalsATS.202005-427RL

138 Cummings MJ, Baldwin MR, Abrams D, et al. Epidemiology, clinical course, and outcomes of critically ill adults with COVID-19 in New York City: a prospective cohort study. Lancet 2020;395:1763-70. doi:10.1016/S0140-6736(20)31189-2

139 Auld SC, Caridi-Scheible M, Blum IM, et al, Emory COVID-19 Quality and Clinical Research Collaborative. ICU and ventilator mortality among critically ill adults with coronavirus disease 2019. Crit Care Med 2020;48:e799-804. doi:10.1097/CCM.0000000000004687

140 Ziehr DR, Alladina J, Petri CR, et al. Respiratory pathophysiology of mechanically ventilated patients with covid-19: a cohort study. Am J Respir Crit Care Med 2020;201:1560-4. doi:10.1164/rccm.2020041163LE

141 Bhatraju PK, Ghassemieh BJ, Nichols M, et al. Covid-19 in critically ill patients in the Seattle region-case series. N Engl) Med 2020;382:2012-22. doi:10.1056/NEIMoa2004500

142 Bellani G, Laffey JG, Pham T, et al, LUNG SAFE Investigators, ESICM Trials Group. Epidemiology, patterns of care, and mortality for patients with acute respiratory distress syndrome in intensive care units in 50 countries. JAMA 2016;315:788-800. doi:10.1001/ jama.2016.0291

143 Grasselli G, Tonetti T, Protti A, et al. Pathophysiology of COVID19-associated acute respiratory distress syndrome: a multicentre prospective observational study. Lancet Respir Med 2020;8:1201-8.

144 Grieco DL, Bongiovanni F, Chen L, et al. Respiratory physiology of COVID-19-induced respiratory failure compared to ARDS of other etiologies. Crit Care 2020;24:529. doi:10.1186/s13054-02003253-2

145 Haudebourg A-F, Perier F, Tuffet S, et al. Respiratory mechanics of covid-19-versus non-covid-19-associated acute respiratory distress syndrome. Am J Respir Crit Care Med 2020;202:287-90. doi:10.1164/rccm.202004-1226LE

146 Brault C, Zerbib Y, Kontar L, et al. covid-19-versus non-covid19-related acute respiratory distress syndrome: differences and similarities. Am J Respir Crit Care Med 2020;202:1301-4. doi:10.1164/rccm.202005-2025LE

147 Hyman JB, Leibner ES, Tandon P, et al. Timing of intubation and in-hospital mortality in patients with coronavirus disease 2019. Crit Care Explor 2020;2:e0254. doi:10.1097/CCE.0000000000000254

148 Hernandez-Romieu AC, Adelman MW, Hockstein MA, et al, Emory COVID-19 Quality and Clinical Research Collaborative. Timing of intubation and mortality among critically ill coronavirus disease 2019 patients: a single-center cohort study. Crit Care Med 2020;48:e1045-53. doi:10.1097/CCM.0000000000004600

149 Wunsch $\mathrm{H}$. Mechanical ventilation in covid-19: interpreting the current epidemiology. Am J Respir Crit Care Med 2020;202:1-4. doi:10.1164/rccm.202004-1385ED

150 Martin-Villares C, Perez Molina-Ramirez C, Bartolome-Benito M, Bernal-Sprekelsen M, COVID ORL ESP Collaborative Group $\left(^{*}\right.$. Outcome of 1890 tracheostomies for critical COVID-19 patients: a national cohort study in Spain. Eur Arch Otorhinolaryngol 2020;4:18. doi:10.1007/s00405-020-06220-3

151 Lu W, Liu X, Wang T, et al. Elevated MUC1 and MUC5AC mucin protein levels in airway mucus of critical ill covid-19 patients. J Med Virol 2020. doi:10.1002/jmv.26406

152 Pinciroli R, Mietto C, Piriyapatsom A, et al. Endotracheal tubes cleaned with a novel mechanism for secretion removal: a randomized controlled clinical study. Respir Care 2016;61:1431-9. doi:10.4187/ respcare. 04363

153 Luo M, Mei Z, Wei L, Cao S, Su S, Wang Y. Precautions for weaning from invasive mechanical ventilation with critically ill COVID-19. Heart Lung 2020;49:869-71. doi:10.1016/j.hrtlng.2020.07.005

154 D'Silva DF, McCulloch TJ, Lim JS, Smith SS, Carayannis D. Extubation of patients with COVID-19. Br J Anaesth 2020;125:e192-5. doi:10.1016/j.bja.2020.03.016 
155 Tobin MJ, Amal J. Weaning from mechanical ventilation. Principles and practice of mechanical ventilation. McGraw-Hill Education, 2013.

156 Abe T, Madotto F, Pham T, et al, LUNG-SAFE Investigators and the ESICM Trials Group. Epidemiology and patterns of tracheostomy practice in patients with acute respiratory distress syndrome in ICUs across 50 countries. Crit Care 2018;22:195. doi:10.1186/s13054018-2126-6

157 Chen W-Q, Ling W-H, Lu C-Y, et al. Which preventive measures might protect health care workers from SARS?BMC Public Health 2009;9:81. doi:10.1186/1471-2458-9-81

158 Botti C, Lusetti F, Peroni S, et al. The role of tracheotomy and timing of weaning and decannulation in patients affected by severe covid-19. Ear Nose Throat J 2020;9:145561320965196.

159 Avilés-Jurado FX, Prieto-Alhambra D, González-Sánchez N, et al. Timing, complications, and safety of tracheotomy in critically ill patients with covid-19. JAMA Otolaryngol Head Neck Surg 2020;147:41-8. doi:10.1001/jamaoto.2020.3641

160 Meister KD, Pandian V, Hillel AT, et al. Multidisciplinary safety recommendations after tracheostomy during covid-19 pandemic: state of the art review. Otolaryngol Head Neck Surg 2020;194599820961990. doi:10.1177/0194599820961990

161 Rovira A, Dawson D, Walker A, et al. Tracheostomy care and decannulation during the covid-19 pandemic. A multidisciplinary clinical practice guideline. Eur Arch Otorhinolaryngol 2020;17:1-9. doi:10.1007/s00405-020-06126-0

162 Lundgren JD, Grund B, Barkauskas CE, et al, ACTIV-3/TICO LY-CoV555 Study Group. A Neutralizing monoclonal antibody for hospitalized patients with covid-19. N Engl J Med 2020. doi:10.1056/ NEJMoa2033130

163 US Food and drug Administration. Emergency use authorization (EUA) of bamlanivimab. 2020. https://wwwfdagov/media/143603/ download.

164 Sterne JAC, Murthy S, Diaz JV, et al, WHO Rapid Evidence Appraisal for COVID-19 Therapies (REACT) Working Group. Association between administration of systemic corticosteroids and mortality among critically ill patients with covid-19: a meta-analysis. JAMA 2020;324:1330-41. doi:10.1001/jama.2020.17023

165 Horby P, Lim WS, Emberson JR, et al, RECOVERY Collaborative Group. Dexamethasone in hospitalized patients with covid-19-preliminary report. N Engl I Med 2020. doi:10.1056/NEJMoa2021436

166 Eastman RT, Roth JS, Brimacombe KR, et al. Remdesivir: A review of its discovery and development leading to emergency use authorization for treatment of covid-19. ACS Cent Sci 2020;6:67283. doi:10.1021/acscentsci.0c00489

167 Beigel JH, Tomashek KM, Dodd LE, et al, ACTT-1 Study Group Members. Remdesivir for the treatment of covid-19-final report. $N$ Engl I Med 2020;383:1813-26. doi:10.1056/NEJMoa2007764

168 Pan H, Peto R, Karim QA, et al. Repurposed antiviral drugs for covid-19 -interim WHO SOLIDARITY trial results.medRxiv [Preprint] 2020:2020. doi:10.15.20209817

169 Spinner CD, Gottlieb RL, Criner GJ, et al, GS-US-540-5774 Investigators. Effect of remdesivir vs standard care on clinical status at 11 days in patients with moderate covid-19: a randomized clinical trial. JAMA 2020;324:1048-57. doi:10.1001/jama.2020.16349

170 Wang Y, Zhang D, Du G, et al. Remdesivir in adults with severe COVID-19: a randomised, double-blind, placebo-controlled, multicentre trial. Lancet 2020;395:1569-78. doi:10.1016/S01406736(20)31022-9

171 Goldman JD, Lye DCB, Hui DS, et al, GS-US-540-5773 Investigators. Remdesivir for 5 or 10 days in patients with severe covid-19. N Engl I Med 2020:383:1827-37. doi:10.1056/NEIMoa2015301

172 Guaraldi G, Meschiari M, Cozzi-Lepri A, et al. Tocilizumab in patients with severe COVID-19: a retrospective cohort study. Lancet Rheumatol 2020;2:e474-84. doi:10.1016/S26659913(20)30173-9

173 Klopfenstein T, Zayet S, Lohse A, et al, HNF Hospital Tocilizumab multidisciplinary team. Tocilizumab therapy reduced intensive care unit admissions and/or mortality in COVID-19 patients. Med Mal Infect 2020;50:397-400. doi:10.1016/j.medmal.2020.05.001

174 Luo P, Liu Y, Qiu L, Liu X, Liu D, Li J. Tocilizumab treatment in COVID-19: A single center experience. J Med Virol 2020;92:814-8. doi:10.1002/jmv.25801

175 Hermine O, Mariette X, Tharaux P-L, Resche-Rigon M, Porcher R, Ravaud P, CORIMUNO-19 Collaborative Group. Effect of tocilizumab vs usual care in adults hospitalized with covid-19 and moderate or severe pneumonia: a randomized clinical trial. JAMA Intern Med 2021;181:32-40. doi:10.1001/jamainternmed.2020.6820

176 Rosas I, Bräu N, Waters M, et al. Tocilizumab in hospitalized patients with covid-19 pneumonia. medRxiv [Preprint] 2020:2020.08.27.20183442. doi:10.1101/2020.08.27.20183442

177 Salvarani C, Dolci G, Massari M, et al, RCT-TCZ-COVID-19 Study Group. Effect of tocilizumab vs standard care on clinical worsening in patients hospitalized with covid-19 pneumonia: a randomized clinical trial. JAMA Intern Med 2021;181:24-31. doi:10.1001/ jamainternmed.2020.6615

178 Stone JH, Frigault MJ, Serling-Boyd NJ, et al, BACC Bay Tocilizumab Trial Investigators. Efficacy of tocilizumab in patients hospitalized with covid-19. N Engl J Med 2020;383:2333-44. doi:10.1056/ NEJMoa2028836

179 Salama C, Han J, Yau L, et al. Tocilizumab in patients hospitalized with covid-19 pneumonia. N Engl / Med 2021;384:20-30. doi:10.1056/ NEJMoa2030340

180 REMAP-CAP Investigators. Gordon AC. Interleukin-6 receptor antagonists in critically ill patients with covid-19-preliminary report.MedRxiv [Preprint] 2021. https://www.medrxiv.org/ content/10.1101/2021.01.07.21249390v1.

181 Veiga VC, Pratts JAGG, Farias DLC, et al. Coalition covid-19 Brazil VI Investigators. Effect of tocilizumab on clinical outcomes at 15 days in patients with severe or critical coronavirus disease 2019: randomised controlled trial. BMJ 2021;372.

182 Horby PW, Pessoa-Amorim G, Peto L, et al. Tocilizumab in patients admitted to hospital with COVID-19 (RECOVERY) preliminary results of a randomised, controlled, open-label, platform trial.medRxiv [Preprint] 2021. https://www.medrxiv.org/ content/10.1101/2021.0211.21249258v1

183 Casadevall A, Dadachova E, Pirofski LA. Passive antibody therapy for infectious diseases. Nat Rev Microbiol 2004;2:695-703. doi:10.1038/nrmicro974

184 Joyner MJ, Senefeld JW, Klassen SA, et al. Effect of convalescent plasma on mortality among hospitalized patients with covid-19: Initial three-month experience. medRxiv [Preprint] 2020:2020.08.12.20169359. doi:10.1101/2020.08.12.20169359

185 Klassen SA, Senefeld JW, Johnson PW, et al. Evidence favoring the efficacy of convalescent plasma for covid-19 therapy.medRxiv [Preprint] 2020:2020.07.29.20162917. doi:10.1101/2020.07.29.20162917

186 Libster R, Perez Marc G, Wappner D, et al. Prevention of severe COVID-19 in the elderly by early high-titer plasma. medRxiv [Preprint] 2021. https://www.medrxiv.org/ content/10.1101/2020.11.20.20234013v1

187 Berger JS, Kunichoff D, Adhikari S, et al. Prevalence and outcomes of D-Dimer elevation in hospitalized patients with covid-19. Arterioscler Thromb Vasc Biol 2020;40:2539-47. doi:10.1161/ ATVBAHA.120.314872

188 National Institutes of Health. NIH ACTIV trial of blood thinners pauses enrollment of critically ill covid-19 patients. 2020. https://www. nih.gov/news-events/news-releases/nih-activ-trial-blood-thinners pauses-enrollment-critically-ill-covid-19-patients

189 National Institutes of Health. Full-dose blood thinners decreased need for life support and improved outcome in hospitalized covid-19 patients. 2021. https://www.nih.gov/news-events/news-releases/ full-dose-blood-thinners-decreased-need-life-support-improvedoutcome-hospitalized-covid-19-patients

190 Herridge MS, Moss M, Hough CL, et al. Recovery and outcomes after the acute respiratory distress syndrome (ARDS) in patients and their family caregivers. Intensive Care Med 2016;42:725-38. doi:10.1007/s00134-016-4321-8

191 Herridge MS, Cheung AM, Tansey CM, et al, Canadian Critical Care Trials Group. One-year outcomes in survivors of the acute respiratory distress syndrome. N Engl J Med 2003;348:683-93. doi:10.1056/ NEJMoa022450

192 Bakhru RN, Davidson JF, Bookstaver RE, et al. Physical function impairment in survivors of critical illness in an ICU Recovery Clinic. J Crit Care 2018:45:163-9. doi:10.1016/j.jcrc.2018.02.001

193 Fan E, Dowdy DW, Colantuoni E, et al. Physical complications in acute lung injury survivors: a two-year longitudinal prospective study. Crit Care Med 2014:42:849-59. doi:10.1097/ CCM.0000000000000040

194 Needham DM, Wozniak AW, Hough CL, et al, National Institutes of Health NHLBI ARDS Network. Risk factors for physical impairment after acute lung injury in a national, multicenter study. Am I Respir Crit Care Med 2014;189:1214-24. doi:10.1164/rccm.201401-01580C

195 Carfi A, Bernabei R, Landi F, Gemelli Against COVID-19 Post-Acute Care Study Group. Group ftGAC-P-ACS. Persistent symptoms in patients after acute covid-19. JAMA 2020;324:603-5. doi:10.1001/ jama.2020.12603

196 Desai SV, Law TJ, Needham DM. Long-term complications of critical care. Crit Care Med 2011;39:371-9. doi:10.1097/ cCM.0b013e3181fd66e 5

197 Bienvenu OJ, Friedman LA, Colantuoni E, et al. Psychiatric symptoms after acute respiratory distress syndrome: a 5-year longitudinal study. Intensive Care Med 2018;44:38-47. doi:10.1007/s00134-017 5009-4

198 Harvey MA, Davidson JE. Postintensive care syndrome: right care, right now...and later. Crit Care Med 2016;44:381-5. doi:10.1097/ CCM.0000000000001531 
199 Galea S, Merchant RM, Lurie N. The mental health consequences of covid-19 and physical distancing: the need for prevention and early intervention. JAMA Intern Med 2020;180:817-8. doi:10.1001/ jamainternmed.2020.1562

200 Mazza MG, De Lorenzo R, Conte C, et al, COVID-19 BioB Outpatient Clinic Study group. Anxiety and depression in COVID-19 survivors: Role of inflammatory and clinical predictors. Brain Behav Immun 2020;89:594-600. doi:10.1016/j.bbi.2020.07.037

201 Zhao YM, Shang YM, Song WB, et al. Follow-up study of the pulmonary function and related physiological characteristics of COVID-19 survivors three months after recovery. EClinicalMedicine 2020;25:100463. doi:10.1016/i. eclinm.2020.100463

202 Liu C, Ye L, Xia R, et al. Chest computed tomography and clinical follow-up of discharged patients with covid-19 in Wenzhou City, Zhejiang, China. Ann Am Thorac Soc 2020;17:1231-7. doi:10.1513/ AnnalsATS.202004-3240C

203 Puntmann VO, Carerj ML, Wieters I, et al. Outcomes of cardiovascular magnetic resonance imaging in patients recently recovered from coronavirus disease 2019 (covid-19). JAMA Cardiol 2020;5:126573. doi:10.1001/jamacardio.2020.3557

204 Rajpal S, Tong MS, Borchers J, et al. Cardiovascular magnetic resonance findings in competitive athletes recovering from covid-19 infection. JAMA Cardiol 2021;6:116-8.

205 Zubair AS, McAlpine LS, Gardin T, Farhadian S, Kuruvilla DE, Spudich S. Neuropathogenesis and neurologic manifestations of the coronaviruses in the age of coronavirus disease 2019: a review. JAMA Neurol 2020;77:1018-27. doi:10.1001/jamaneurol.2020.2065

206 Ellul MA, Benjamin L, Singh B, et al. Neurological associations of COVID-19. Lancet Neurol 2020;19:767-83. doi:10.1016/S1474 4422(20)30221-0

207 Lambert NJ, Corps S. Covid-19 “long hauler” symptoms survey report. Indiana University School of Medicine. 2020. https://dig.abclocal. go.com/wls/documents/2020/072720-wls-covid-symptom-studydoc.pdf

208 Pandharipande PP, Girard TD, Jackson JC, et al, BRAIN-ICU Study Investigators. Long-term cognitive impairment after critical illness. N Engl J Med 2013;369:1306-16. doi:10.1056/NEJMoa1301372

209 Greenhalgh T, Knight M, A'Court C, Buxton M, Husain L. Management of post-acute covid-19 in primary care. BMJ 2020;370:m3026. doi:10.1136/bmj.m3026

210 Tenforde MW, Kim SS, Lindsell CJ, et al, IVY Network Investigators, CDC COVID-19 Response Team, IVY Network Investigators. Symptom duration and risk factors for delayed return to usual health among outpatients with covid-19 in a multistate health care systems network-United States, March-June 2020. MMWR Morb Mortal Wkly Rep 2020;69:993-8. doi:10.15585/mmwr.mm6930e1

211 Halpin SJ, Mclvor C, Whyatt G, et al. Postdischarge symptoms and rehabilitation needs in survivors of COVID-19 infection: A cross-sectional evaluation. J Med Virol 2021;93:1013-22. doi:10.1002/jmv.26368

212 Needham DM, Davidson J, Cohen $\mathrm{H}$, et al. Improving long-term outcomes after discharge from intensive care unit: report from a stakeholders' conference. Crit Care Med 2012;40:502-9. doi:10.1097/CCM.0b013e318232da75

213 Griffiths J, Hatch RA, Bishop J, et al. An exploration of social and economic outcome and associated health-related quality of life after critical illness in general intensive care unit survivors: a 12-month follow-up study. Crit Care 2013;17:R100. doi:10.1186/cc12745

214 Sese D, Biehl M. Post intensive care syndrome (PICS) in the time of covid-19-implications post pandemic. CCJM Curbside Consults, 2020, doi:10.3949/ccjm.87a.ccc055

215 Montauk TR, Kuhl EA. COVID-related family separation and trauma in the intensive care unit. Psychol Trauma 2020;12(S1):S96-7. doi: $10.1037 /$ tra0000839

216 Marra A, Ely EW, Pandharipande PP, Patel MB. The ABCDEF bundle in critical care. Crit Care Clin 2017;33:225-43. doi:10.1016/j. ccc.2016.12.005

217 Devlin JW, O'Neal HRJr, Thomas C, et al. Strategies to optimize ICU liberation (A to F) bundle performance in critically ill adults with coronavirus disease 2019. Crit Care Explor 2020;2:e0139. doi:10.1097/CCE.0000000000000139

218 Devlin JW, Skrobik Y, Gélinas C, et al. Clinical practice guidelines for the prevention and management of pain, agitation/sedation, delirium, immobility, and sleep disruption in adult patients in the ICU. Crit Care Med 2018;46:e825-73. doi:10.1097/ CCM.0000000000003299

219 Hodgson CL, Bailey M, Bellomo R, et al, Trial of Early Activity and Mobilization Study Investigators. A binational multicenter pilot feasibility randomized controlled trial of early goal-directed mobilization in the ICU. Crit Care Med 2016;44:1145-52. doi:10.1097/CCM.0000000000001643

220 McWilliams DJ, Atkinson D, Carter A, Foëx BA, Benington $\mathrm{S}$, Conway DH. Feasibility and impact of a structured, exercise-based rehabilitation programme for intensive care survivors. Physiother Theory Pract 2009;25:566-71. doi:10.3109/09593980802668076

221 Elliott D, McKinley S, Alison JA, Aitken LM, King MT. Study protocol: home-based physical rehabilitation for survivors of a critical illness [ACTRN12605000166673][ACTRN12605000166673]. Crit Care 2006;10:R90. doi:10.1186/cc4949

222 Ullman AJ, Aitken LM, Rattray J, et al. Intensive care diaries to promote recovery for patients and families after critical illness: A Cochrane Systematic Review. Int J Nurs Stud 2015;52:1243-53. doi:10.1016/j.ijnurstu.2015.03.020

223 Jones C, Bäckman C, Capuzzo M, et al, RACHEL group. Intensive care diaries reduce new onset post traumatic stress disorder following critical illness: a randomised, controlled trial. Crit Care 2010;14:R168. doi:10.1186/cC9260

224 Peris A, Bonizzoli M, Iozzelli D, et al. Early intra-intensive care unit psychological intervention promotes recovery from post traumatic stress disorders, anxiety and depression symptoms in critically ill patients. Crit Care 2011;15:R41. doi:10.1186/cc10003

225 Hosey MM, Jaskulski J, Wegener ST, Chlan LL, Needham DM. Animal-assisted intervention in the ICU: a tool for humanization. Crit Care 2018;22:22. doi:10.1186/s13054-018-1946-8

226 McPeake J, Hirshberg EL, Christie LM, et al. Models of peer support to remediate post-intensive care syndrome: a report developed by the Society of Critical Care Medicine Thrive International Peer Support Collaborative. Crit Care Med 2019;47:e21-7. doi:10.1097/ CCM.0000000000003497

227 Haines KJ, Beesley SJ, Hopkins RO, et al. Peer support in critical care: a systematic review. Crit Care Med 2018;46:1522-31. doi:10.1097/ CCM.0000000000003293

228 Mayer KP, Sturgill JL, Kalema AG, et al. Recovery from COVID-19 and acute respiratory distress syndrome: the potential role of an intensive care unit recovery clinic: a case report. / Med Case Rep 2020;14:161. doi:10.1186/s13256-020-02481-y

229 Sevin CM, Bloom SL, Jackson JC, Wang L, Ely EW, Stollings JL. Comprehensive care of ICU survivors: Development and implementation of an ICU recovery center. J Crit Care 2018;46:141-8. doi:10.1016/j.jcrc.2018.02.011

230 Haines KJ, Sevin CM, Hibbert E, et al. Key mechanisms by which postICU activities can improve in-ICU care: results of the international THRIVE collaboratives. Intensive Care Med 2019;45:939-47. doi:10.1007/s00134-019-05647-5

231 Del Rio C, Collins LF, Malani P. Long-term health consequences of covid-19. JAMA 2020;324:1723-4. doi:10.1001/jama.2020.19719

232 Bhimraj A, Morgan RL, Shumaker AH, et al. IDSA guidelines on the treatment and management of patients with covid-19. Clin Infect Dis 2021. https://www.idsociety.org/practice-guideline/covid-19guideline-treatment-and-management/

233 Hanson KE, Caliendo A, Arias C, et al. IDSA guidelines on the diagnosis of covid-19: molecular diagnostic testing. Clin Infect Dis 2020. https://www.idsociety.org/practice-guideline/covid-19. guideline-diagnostics/

234 World Health Organization. Country \& Technical GuidanceCoronavirus disease (COVID-19). 2021. https://www.who.int/ emergencies/diseases/novel-coronavirus-2019/technical-guidancepublications.

235 Society of Critical Care Medicine. Guidelines on the management of critically ill adults with coronavirus disease 2019 (COVID-19). 2021. https://www.sccm.org/SurvivingSepsisCampaign/Guidelines/ COVID-19

236 National Institutes of Health. COVID-19 treatment guidelines. Clinical spectrum of SARS-CoV-2 infection. 2020. https://www. covid19treatmentguidelinesnihgov/overview/clinical-spectrum 